\title{
Non Separable Lifting Scheme with Adaptive Update Step for Still and Stereo Image Coding
}

\author{
M. Kaaniche ${ }^{\mathrm{a}, \mathrm{b}}$, A. Benazza-Benyahia ${ }^{\mathrm{b}}$, B. Pesquet-Popescu ${ }^{\mathrm{a}}$, J.- C. Pesquet ${ }^{\mathrm{c}}$ \\ ${ }^{a}$ Telecom ParisTech, Signal and Image Proc. Dept. 37-39, rue Dareau, 75014 Paris, France \\ ${ }^{b}$ SUP'COM, URISA, Cité Technologique des Communications, 2083, Tunisia \\ ${ }^{c}$ Université Paris-Est, IGM and UMR-CNRS 8049, Champs-sur-Marne, 77454 \\ Marne-la-Vallée, France
}

\begin{abstract}
Many existing works related to lossy-to-lossless multiresolution image compression are based on the lifting concept. It is worth noting that a separable lifting scheme may not appear very efficient to cope with the two-dimensional characteristics of edges which are neither horizontal nor vertical. In this paper, we propose to use $2 D$ non separable lifting schemes that still enable progressive reconstruction and exact decoding of images. Their relevant advantage is to yield a tractable optimization of all the involved decomposition operators. More precisely, we design the prediction operators by minimizing the variance of the detail coefficients. Concerning the update filters, we propose a new optimization criterion which aims at reducing the inherent aliasing artifacts. A theoretical analysis of the proposed method is conducted in terms of the adaptation criterion considered in the optimization of the update filter. Simulations carried out on still images and residual ones generated from stereo pairs show the benefits which can be drawn from the proposed optimization of the lifting operators.
\end{abstract}

Key words: lossless compression, progressive reconstruction, lifting schemes, separable transforms, non separable transforms, adaptive transforms, multiresolution analysis, wavelets, stereo coding.

\footnotetext{
Email addresses: kaaniche@telecom-paristech.fr (M. Kaaniche), benazza.amel@supcom.rnu.tn (A. Benazza-Benyahia), pesquet@telecom-paristech.fr (B. Pesquet-Popescu), jean-christophe.pesquet@univ-paris-est.fr (J.- C. Pesquet)
} 


\section{Introduction}

During the two past decades, much attention has been paid to the theory of filter banks and subband coding. Very efficient compression methods for still images can be built, which take advantage of multiresolution representation features, good energy compaction and decorrelation properties $[1,2]$. Image coding techniques have been extensively studied for various application requirements [3, 4]. For instance, lossless compression techniques are required in some fields like remote sensing imaging or medical imaging for which any distortion in the reconstructed image may lead to an erroneous interpretation of the image [5]. Moreover, the scalability property is a highly desirable functionality for telebrowsing applications since the refinement of the decoded image depends on the user needs and/or the available network bandwidth. The challenge in many lossy-to-lossless coding schemes is the design of a sparse multiresolution representation of the original image. Lifting Schemes (LS) have proved to be efficient tools for this purpose $[6,7]$. A generic LS applied to a 1D signal consists of three modules referred to as split, predict and update. In the first step, the even indexed samples are separated from the odd indexed ones. Then, each sample of one of the two resulting subsets (say the even one) is predicted from the odd indexed samples and a prediction error or detail coefficient is computed. Finally, the update step generates a coarser approximation of the initial signal thanks to a smoothing of the odd indexed samples based on the detail coefficients. Generally, for 2D signals, the LS is handled in a separable way by cascading 1D LS along the horizontal direction, then along the vertical one. It is worth noting that a separable LS may not appear very efficient to cope with the two-dimensional characteristics of edges which are neither horizontal nor vertical [8]. In addition to monocular images, LS have been extensively used for stereo image coding $[9,10]$. Indeed, most of the existing works rely on disparity compensation techniques due to their similarity with the motion compensation techniques which are popular for video coding [11]. The first step in this approach consists of estimating the disparity map. Then, one image is considered as a reference image and the other is predicted in order to generate a prediction error referred to as a residual image. Finally, the disparity field, the reference image and the residual one are encoded [10, 12]. In this context, Moellenhoff and Maier [13] analyzed the characteristics of the residual image and proved that such images have properties 
different from natural images. This suggests that transforms working well for natural images may not be as well-suited for residual images.

These shortcomings have motivated the development of several alternatives in order to improve the coding efficiency of lifting-based coding methods. The reported solutions fall into the two following categories:

- A first approach consists of designing Non Separable Lifting Schemes (NSLS) in order to better capture the actual two-dimensional contents of the image $[8,14,15$, $16,17]$.

- A second alternative aims at adapting the operators of the conventional separable lifting scheme to the characteristics of the input image [18]-[31].

In the first approach, instead of using samples from the same rows (resp. columns) while processing the image along the lines (resp. columns), 2D NSLS provide more choices in the selection of the samples by using horizontal, vertical and oblique directions at the prediction step [8]. Quincunx lifting schemes constitute another example of NSLS which was proposed in the context of image coding $[14,15]$. It is worth mentioning that the $2 \mathrm{D}$ operators in a NSLS can satisfy some appealing properties (e.g. orthogonality) unlike those related to a separable LS [16]. The orthogonality property may be interesting in the framework of image coding since the mean square reconstruction error in the spatial domain is then equal to the mean square quantization error of the wavelet coefficients. Furthermore, in [17], 2D NSLS were proposed with a reduced number of rounding operations w.r.t. their equivalent separable LS. Therefore, the coding performance can be improved both in the lossless and the lossy modes.

In parallel to these works on NSLS, a second approach was also investigated in order to exploit the directional correlation in the image by the operators involved in a separable LS. To the best of our knowledge, most existing works have mainly focused on the optimization of the prediction operators. For instance, Gerek and al. [19] proposed a 2D edge-adaptive lifting scheme by considering three direction angles of prediction $\left(0^{\circ}, 45^{\circ}\right.$ and $\left.135^{\circ}\right)$ and by selecting the orientation which leads to the minimum prediction error. In [20], three separable prediction filters with different numbers of vanishing moments are employed, and then the best prediction is chosen according to the local fea- 
tures. Recently, Ding et al. [21] have built an adaptive directional lifting structure with perfect reconstruction: the prediction is performed in local windows in the direction of high pixel correlation. A good directional resolution is achieved by employing fractional pixel precision level. A similar approach was also adopted in [22]. An improvement was proposed in [23] thanks to a directional adaptive interpolation. In [25], the prediction filter is optimized according to the image statistics based on the value of the conditional probability density function (pdf) at the current sample to be predicted given its two neighbors. Consequently, an estimation of the pdf should be available at the coder and the decoder side. Note that the main drawback of this method as well as directional wavelet transform ones $[21,22,23,24,26]$ is that they require to transmit losslessly a side information to the decoder which may affect the whole compression performance especially at low bitrates. Furthermore, such adaptive methods lead to an increase of the computational load required for the selection of the best direction of prediction. Besides, it can be noticed that there are few works which have discussed the problem of the update filter. Among these works, in [27, 28], the update operator of a separable LS is adaptively computed thanks to a non linear decision rule using the local gradient information. An extension of this method to $2 \mathrm{D}$ non separable schemes that have a spatially adaptive low-pass filter is proposed in [29]. Another alternative is to adapt the update so that the reconstruction error is minimized when the detail coefficients are canceled [30, 31]. Recently, an adaptation procedure was proposed in [32]. It consists of designing an update and prediction Neville filters whose corresponding primal and dual wavelets have the desired number of vanishing moments. The remaining degrees of freedom are used to optimize a criterion depending on the underlying application. In their paper, the authors were interested in finding the wavelet basis that maximizes the retrieval performance for content-based image retrieval applications.

In this paper, we aim at fully exploiting the flexibility of a NSLS through an adaptation of all the involved operators. In that sense, we are interested in combining the advantages of the two aforementioned approaches. Note that adaptive NSLS were already investigated $[15,31,33]$ by putting emphasis on the optimization of the predictor. One of the main contributions of this paper is the optimization of the update filter by using a criterion which allows us to reduce aliasing effects. In this way, we build a fully-optimized NSLS 
which is better adapted to the characteristics of the input image. Moreover, we show that the proposed optimization method leads to the same optimal update filter when the optimization is performed either before or after the decimation step. Another major contribution of our work consists of deriving theoretical expressions of the optimal filter coefficients based on the correlation factors of the input field. These expressions may be interesting in the implementation process of the proposed optimization method. An advantage of our adaptive method is that the side information required for the transmission of all the filter coefficients is negligible compared to the global bitrate. Furthermore, our method guarantees a perfect reconstruction of the original image.

The remainder of this paper is organized as follows. In Section 2, a 2D non separable lifting structure is presented and its link with some existing 1D LS is emphasized. In Section 3, we describe the proposed approach for the design of both optimal prediction and update filters. In Section 4, we conduct a theoretical analysis of the proposed method in order to evaluate the gain achieved by the proposed update filter optimization together with a simple statistical modeling of images. We show that in the case of a first order 2D autoregressive model for the input images, the filters can be easily deduced only based on the spatial correlation coefficients. Section 5 discusses the transmission problem of the resulting wavelet coefficients as well as the adaptive filter weights. Finally, in Section 6, experimental results are given and some conclusions are drawn in Section 7.

\section{2D non separable LS structure}

\subsection{Principle}

Let $x$ denote the digital image to be coded. At each resolution level $j$ and each pixel location $(m, n)$, its approximation coefficient is denoted by $x_{j}(m, n)$ and the associated four polyphase components by $x_{0, j}(m, n)=x_{j}(2 m, 2 n), x_{1, j}(m, n)=x_{j}(2 m, 2 n+1)$, $x_{2, j}(m, n)=x_{j}(2 m+1,2 n)$, and $x_{3, j}(m, n)=x_{j}(2 m+1,2 n+1)$. Without loss of generality, we assume that the polyphase components are the input coefficients of the $2 \mathrm{D}$ NSLS described in [17] and depicted in Fig. 1, where $\mathbf{P}_{j}^{(H H)}, \mathbf{P}_{j}^{(L H)}, \mathbf{P}_{j}^{(H L)}$ and $\mathbf{U}_{j}$ represent the four analysis filters employed to generate the detail coefficients $x_{j+1}^{(H H)}$ oriented diagonally, $x_{j+1}^{(L H)}$ oriented vertically, $x_{j+1}^{(H L)}$ oriented horizontally, and the approximation coefficients $x_{j+1}$. It is easy to derive the expressions of the resulting coefficients in the $2 \mathrm{D}$ 


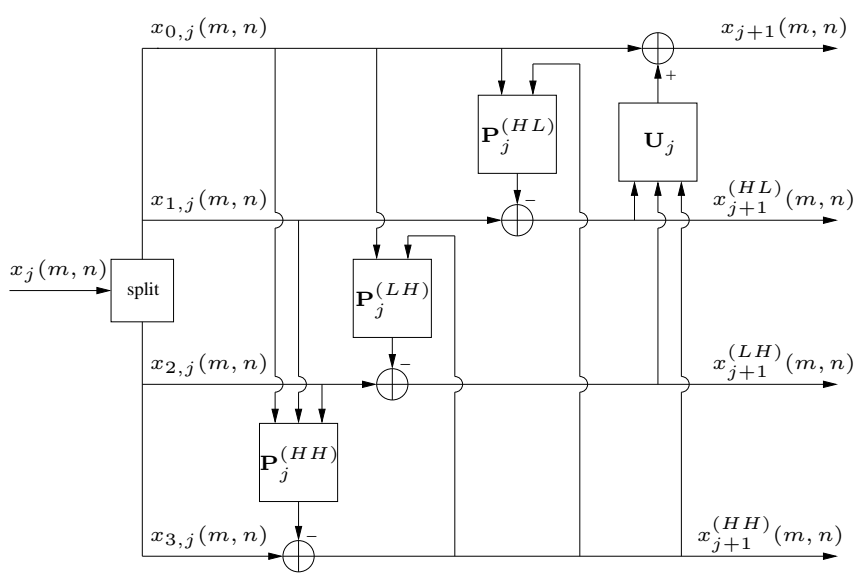

Figure 1: NSLS decomposition structure.

$z$-transform domain ${ }^{1}$. Indeed, the $z$-transforms of the output coefficients can be written as follows:

$$
\begin{aligned}
X_{j+1}^{(H H)}\left(z_{1}, z_{2}\right) & =X_{3, j}\left(z_{1}, z_{2}\right)-\left\lfloor P_{0, j}^{(H H)}\left(z_{1}, z_{2}\right) X_{0, j}\left(z_{1}, z_{2}\right)+P_{1, j}^{(H H)}\left(z_{1}, z_{2}\right) X_{1, j}\left(z_{1}, z_{2}\right)\right. \\
& \left.+P_{2, j}^{(H H)}\left(z_{1}, z_{2}\right) X_{2, j}\left(z_{1}, z_{2}\right)\right\rfloor, \\
X_{j+1}^{(L H)}\left(z_{1}, z_{2}\right)= & X_{2, j}\left(z_{1}, z_{2}\right)-\left\lfloor P_{0, j}^{(L H)}\left(z_{1}, z_{2}\right) X_{0, j}\left(z_{1}, z_{2}\right)+P_{1, j}^{(L H)}\left(z_{1}, z_{2}\right) X_{j+1}^{(H H)}\left(z_{1}, z_{2}\right)\right\rfloor, \\
X_{j+1}^{(H L)}\left(z_{1}, z_{2}\right)= & X_{1, j}\left(z_{1}, z_{2}\right)-\left\lfloor P_{0, j}^{(H L)}\left(z_{1}, z_{2}\right) X_{0, j}\left(z_{1}, z_{2}\right)+P_{1, j}^{(H L)}\left(z_{1}, z_{2}\right) X_{j+1}^{(H H)}\left(z_{1}, z_{2}\right)\right\rfloor, \\
X_{j+1}\left(z_{1}, z_{2}\right)= & X_{0, j}\left(z_{1}, z_{2}\right)+\left\lfloor U_{j}^{(H L)}\left(z_{1}, z_{2}\right) X_{j+1}^{(H L)}\left(z_{1}, z_{2}\right)+U_{j}^{(L H)}\left(z_{1}, z_{2}\right) X_{j+1}^{(L H)}\left(z_{1}, z_{2}\right)\right. \\
& \left.+U_{j}^{(H H)}\left(z_{1}, z_{2}\right) X_{j+1}^{(H H)}\left(z_{1}, z_{2}\right)\right\rfloor
\end{aligned}
$$

where, for every $i \in\{0,1,2\}$ and $o \in\{H H, H L, L H\}$,

$$
\begin{aligned}
P_{i, j}^{(o)}\left(z_{1}, z_{2}\right) & =\sum_{(k, l) \in \mathcal{P}_{i, j}^{(o)}} p_{i, j}^{(o)}(k, l) z_{1}^{-k} z_{2}^{-l}, \\
U_{j}^{(o)}\left(z_{1}, z_{2}\right) & =\sum_{(k, l) \in \mathcal{U}_{j}^{(o)}} u_{j}^{(o)}(k, l) z_{1}^{-k} z_{2}^{-l} .
\end{aligned}
$$

\footnotetext{
${ }^{1}$ The $z$-transform of a signal $x$ will be denoted in capital letters by $X$.
} 
The set $\mathcal{P}_{i, j}^{(o)}$ (resp. $\mathcal{U}_{j}^{(o)}$ ) and the coefficients $p_{i, j}^{(o)}(k, l)$ (resp. $\left.u_{j}^{(o)}(k, l)\right)$ denote the support and the weights of the three prediction filters (resp. of the update filter). Note that we introduced the rounding operations $\lfloor$.$\rfloor in order to allow lossy-to-lossless encoding of$ the coefficients. Once the considered NSLS structure has been defined, it may appear instructive to see how it is related to some conventional separable lifting structures.

\subsection{Links with conventional separable lifting structures}

It can be checked that the conventional 5/3 transform and the Haar one are particular cases of the structure illustrated in Fig. 1. For example, for the separable $5 / 3$ transform which was selected for the lossless mode of the JPEG2000 standard [4], we get:

$$
\begin{aligned}
P_{0, j}^{(H H)}\left(z_{1}, z_{2}\right) & =-\frac{1}{4}\left(1+z_{1}+z_{2}+z_{1} z_{2}\right), \\
P_{1, j}^{(H H)}\left(z_{1}, z_{2}\right) & =\frac{1}{2}\left(1+z_{1}\right), P_{2, j}^{(H H)}\left(z_{1}, z_{2}\right)=\frac{1}{2}\left(1+z_{2}\right), \\
P_{0, j}^{(L H)}\left(z_{1}, z_{2}\right) & =\frac{1}{2}\left(1+z_{1}\right), P_{1, j}^{(L H)}\left(z_{1}, z_{2}\right)=-\frac{1}{4}\left(1+z_{2}^{-1}\right), \\
P_{0, j}^{(H L)}\left(z_{1}, z_{2}\right) & =\frac{1}{2}\left(1+z_{2}\right), P_{1, j}^{(H L)}\left(z_{1}, z_{2}\right)=-\frac{1}{4}\left(1+z_{1}^{-1}\right), \\
U_{j}^{(H L)}\left(z_{1}, z_{2}\right) & =\frac{1}{4}\left(1+z_{1}^{-1}\right), U_{j}^{(L H)}\left(z_{1}, z_{2}\right)=\frac{1}{4}\left(1+z_{2}^{-1}\right), \\
U_{j}^{(H H)}\left(z_{1}, z_{2}\right) & =-\frac{1}{16}\left(1+z_{1}^{-1}+z_{2}^{-1}+z_{1}^{-1} z_{2}^{-1}\right) .
\end{aligned}
$$

After similar manipulations, it is possible to check that any separable predict-update LS (P-U structure) as those tabulated in [7] has an equivalent 2D-NSLS counterpart [17]. Subsequently, we will show that this equivalence facilitates the derivation of adaptive extensions of the associated operators. Indeed, in a coding framework, the sparseness of any LS-based multiresolution representation clearly depends on the choice of the prediction and update operators. In the next section, we address the issue of an optimal design of the NSLS operators for coding purposes.

\section{Proposed optimization method}

In what follows, for the sake of simplicity, we assume that the initial image $x$ (after subtracting its mean value) is modeled as the realization of a zero-mean wide-sense stationary random process. If we omit the rounding operations, at a given resolution 
level $j$, the approximation image $x_{j}$ is also the realization of a $2 \mathrm{D}$ wide sense stationary process with autocorrelation function $R_{x_{j}}$ :

$$
\forall(k, l) \in \mathbb{Z}^{2}, \quad R_{x_{j}}(k, l)=\mathrm{E}\left[x_{j}(m, n) x_{j}(m-k, n-l)\right] .
$$

\subsection{Optimization of the predictors}

Since the detail coefficients are defined as prediction errors, the prediction operators can be optimized so as to minimize the variance of the coefficients at each resolution level. The rounding operators being omitted, it is readily shown that the minimum variance predictors must satisfy the well-known Yule-Walker equations. For example, for the prediction vector $\mathbf{p}_{j}^{(H H)}$, the normal equations read

$$
\mathrm{E}\left[\tilde{\mathbf{x}}_{j}(m, n) \tilde{\mathbf{x}}_{j}(m, n)^{\top}\right] \mathbf{p}_{j}^{(H H)}=\mathrm{E}\left[x_{3, j}(m, n) \tilde{\mathbf{x}}_{j}(m, n)\right]
$$

where

- $\mathbf{p}_{j}^{(H H)}=\left(\mathbf{p}_{0, j}^{(H H)}, \mathbf{p}_{1, j}^{(H H)}, \mathbf{p}_{2, j}^{(H H)}\right)^{\top}$ is the prediction vector where, for each $i \in$ $\{0,1,2\}$,

$$
\mathbf{p}_{i, j}^{(H H)}=\left(p_{i, j}^{(H H)}(k, l)\right)_{(k, l) \in \mathcal{P}_{i, j}^{(H H)}},
$$

- $\tilde{\mathbf{x}}_{j}(m, n)=\left(\mathbf{x}_{0, j}(m, n), \mathbf{x}_{1, j}(m, n), \mathbf{x}_{2, j}(m, n)\right)^{\top}$ is the reference vector with

$$
\mathbf{x}_{i, j}(m, n)=\left(x_{i, j}(m-k, n-l)\right)_{(k, l) \in \mathcal{P}_{i, j}^{(H H)}} .
$$

The other optimal prediction filters $\mathbf{p}_{j}^{(H L)}$ and $\mathbf{p}_{j}^{(L H)}$ are obtained in a similar way.

\subsection{Optimization of the update operator}

Optimizing the update operator is less obvious than optimizing the predictions. Recently, an update optimization method has been proposed by Gouze et al. [30, 31]. It consists of designing the update operator in order to minimize a reconstruction error. In other words, the update operator is optimized by minimizing the distortion between the original image $x_{j}$ and the reconstructed image $\hat{x}_{j}$, when the detail coefficients are 
canceled. In the case of the NSLS, this criterion is given by:

$$
\begin{aligned}
\mathcal{J}_{\text {Gouze }} & =\mathrm{E}\left[\left(x_{j}(m, n)-\hat{x}_{j}(m, n)\right)^{2}\right] \\
& =\frac{1}{4}\left(\mathrm{E}\left[\left(x_{0, j}(m, n)-\hat{x}_{0, j}(m, n)\right)^{2}\right]+\mathrm{E}\left[\left(x_{1, j}(m, n)-\hat{x}_{1, j}(m, n)\right)^{2}\right]\right. \\
& \left.+\mathrm{E}\left[\left(x_{2, j}(m, n)-\hat{x}_{2, j}(m, n)\right)^{2}\right]+\mathrm{E}\left[\left(x_{3, j}(m, n)-\hat{x}_{3, j}(m, n)\right)^{2}\right]\right) .
\end{aligned}
$$

Then, the minimization of the criterion (13) provides the optimal update operator. Due to the complexity of the resulting linear system of equations when a NSLS is considered, we will not describe it, the reader being referred to [31] for more details.

In this paper, we propose to employ an optimization criterion, which aims at reducing the inherent aliasing artefacts (see also our preliminary work in [34]). Firstly, it can be noticed that the transfer function of the low-pass filter relating $x_{j}$ to the undecimated version of $x_{j+1}$ can be expressed as

$$
F_{j}\left(z_{1}, z_{2}\right)=1+\sum_{o \in\{H L, L H, H H\}} \sum_{(k, l) \in \mathcal{U}_{j}^{(o)}} u_{j}^{(o)}(k, l) z_{1}^{-2 k} z_{2}^{-2 l} A_{j}^{(o)}\left(z_{1}, z_{2}\right)
$$

where $A_{j}^{(o)}\left(z_{1}, z_{2}\right)$ is a transfer function which depends on the prediction coefficients. Since $F_{j}$ aims at computing a smooth version of the image $x_{j}$, we propose to design the update filter in order to reduce the aliasing effects. More precisely, we adopt a new criterion $\tilde{\mathcal{J}}$ which accounts for the difference between the output of the $F_{j}$ filter and the output of an ideal low-pass filter

$$
\tilde{\mathcal{J}}\left(u_{j}\right)=\int_{-\pi}^{\pi}\left|F_{j}\left(e^{\imath \omega_{1}}, e^{\imath \omega_{2}}\right)-H\left(e^{\imath \omega_{1}}, e^{\imath \omega_{2}}\right)\right|^{2} S_{x_{j}}\left(\omega_{1}, \omega_{2}\right) d \omega_{1} d \omega_{2}
$$

where $S_{x_{j}}$ is the power spectrum density of $x_{j}$ and $H$ denotes the transfer function of the ideal filter ( $h$ will subsequently designate its impulse response). Note that $\tilde{\mathcal{J}}$ is not limited to a simple quadratic mean square error between the update filter and the ideal low-pass filter: the proposed criterion exploits the characteristics of the input signals, so making the method adaptive. By cancelling the gradient of $\tilde{\mathcal{J}}$, the optimal update 
weights satisfy, for each $\left(k^{\prime}, l^{\prime}\right) \in \mathcal{U}_{j}^{\left(o^{\prime}\right)}$ and $o^{\prime} \in\{H L, L H, H H\}$, the following condition:

$$
\begin{gathered}
\sum_{o \in\{H L, L H, H H\}} \sum_{(k, l) \in \mathcal{U}_{j}^{(o)}} u_{j}^{(o)}(k, l) \sum_{(p, q)} \sum_{\left(p^{\prime}, q^{\prime}\right)} a_{j}^{(o)}(p, q) a_{j}^{\left(o^{\prime}\right)}\left(p^{\prime}, q^{\prime}\right) \\
\times R_{x_{j}}\left(p^{\prime}-p+2 k^{\prime}-2 k, q^{\prime}-q+2 l^{\prime}-2 l\right) \\
=\sum_{(p, q)} \sum_{\left(p^{\prime}, q^{\prime}\right)} h(p, q) a_{j}^{\left(o^{\prime}\right)}\left(p^{\prime}, q^{\prime}\right) R_{x_{j}}\left(p^{\prime}-p+2 k^{\prime}, q^{\prime}-q+2 l^{\prime}\right) \\
-\sum_{\left(p^{\prime}, q^{\prime}\right)} a_{j}^{\left(o^{\prime}\right)}\left(p^{\prime}, q^{\prime}\right) R_{x_{j}}\left(p^{\prime}+2 k^{\prime}, q^{\prime}+2 l^{\prime}\right) .
\end{gathered}
$$

It must be emphasized that this criterion $\tilde{\mathcal{J}}$ measures the quadratic error between the output of the low-pass filter $F_{j}$ and the ideal one $H$ before decimation. However, it may also be interesting to minimize the error after the decimation step. For this purpose, we propose to use an alternative criterion which measures the error between the decimated versions:

$$
\begin{aligned}
\mathcal{J}\left(u_{j}\right)= & \mathrm{E}\left[\left(x_{j+1}(m, n)-y_{j+1}(m, n)\right)^{2}\right] \\
= & \mathrm{E}\left[\left(x_{0, j}(m, n)+\sum_{o \in\{H L, L H, H H\}} \sum_{(k, l) \in \mathcal{U}_{j}^{(o)}} u_{j}^{(o)}(k, l) x_{j+1}^{(o)}(m-k, n-l)\right.\right. \\
& \left.\left.-y_{j+1}(m, n)\right)^{2}\right]
\end{aligned}
$$

where $y_{j+1}(m, n)=\tilde{y}_{j}(2 m, 2 n)=\left(h * x_{j}\right)(2 m, 2 n)$.

Recall that the $2 \mathrm{D}$ ideal rectangular low-pass filter is defined in the spatial domain by:

$$
\forall(m, n) \in \mathbb{Z}^{2}, \quad h(m, n)=\frac{1}{4} \operatorname{sinc}\left(\frac{m \pi}{2}\right) \operatorname{sinc}\left(\frac{n \pi}{2}\right) .
$$

The optimal update coefficients minimizing the second criterion $\mathcal{J}$ verify, for each $\left(k^{\prime}, l^{\prime}\right) \in$ $\mathcal{U}_{j}^{\left(o^{\prime}\right)}$ and $o^{\prime} \in\{H L, L H, H H\}$, the following equation:

$$
\begin{aligned}
& \sum_{o \in\{H L, L H, H H\}} \sum_{(k, l) \in \mathcal{U}_{j}^{(o)}} u_{j}^{(o)}(k, l) \mathrm{E}\left[x_{j+1}^{(o)}(m-k, n-l) x_{j+1}^{\left(o^{\prime}\right)}\left(m-k^{\prime}, n-l^{\prime}\right)\right] \\
= & \mathrm{E}\left[y_{j+1}(m, n) x_{j+1}^{\left(o^{\prime}\right)}\left(m-k^{\prime}, n-l^{\prime}\right)\right]-\mathrm{E}\left[x_{0, j}(m, n) x_{j+1}^{\left(o^{\prime}\right)}\left(m-k^{\prime}, n-l^{\prime}\right)\right] .
\end{aligned}
$$

It is worth pointing out that the resulting solution (19) is equivalent to the one obtained by minimizing $\tilde{\mathcal{J}}$ (16). Indeed, by using (4) and (14), it can be checked that:

$$
x_{j+1}^{(o)}(m, n)=\sum_{(p, q)} a_{j}^{(o)}(p, q) x_{j}(2 m-p, 2 n-q)
$$


where $a_{j}^{(o)}(p, q)$ are the coefficients of the transfer function $A_{j}^{(o)}\left(z_{1}, z_{2}\right)$. Thus, by substituting this expression into (19), we deduce that the minimizations of criteria $\tilde{\mathcal{J}}$ and $\mathcal{J}$ lead to the same optimal solution.

As computing an error between the decimated versions of the image allows us to reduce the complexity of our optimization method, we propose to use the second criterion $\mathcal{J}$. In this case, it can be noticed that (19) can be rewritten concisely as follows:

$$
\mathrm{E}\left[\mathbf{x}_{j+1}(m, n) \mathbf{x}_{j+1}(m, n)^{\top}\right] \mathbf{u}_{j}=\mathrm{E}\left[y_{j+1}(m, n) \mathbf{x}_{j+1}(m, n)\right]-\mathrm{E}\left[x_{0, j}(m, n) \mathbf{x}_{j+1}(m, n)\right]
$$

where

- $\mathbf{u}_{j}=\left(u_{j}^{(o)}(k, l)\right)_{(k, l) \in \mathcal{U}_{j}^{(o)}, o \in\{H L, L H, H H\}}^{\top}$ is the update weight vector,

- $\mathbf{x}_{j+1}(m, n)=\left(x_{j+1}^{(o)}(m-k, n-l)\right)_{(k, l) \in \mathcal{P}_{i, j}^{(o)}, o \in\{H L, L H, H H\}}^{\top}$ is the update reference vector containing the detail signals.

Consequently, the optimal update weights are solutions of a linear system of equations given the second order characteristics of the involved signals. More specifically, in order to solve (21), we need to compute

- the autocorrelation matrix $\boldsymbol{\Gamma}_{j}=\mathrm{E}\left[\mathbf{x}_{j+1}(m, n) \mathbf{x}_{j+1}(m, n)^{\top}\right]$,

- the cross-correlation vector $\mathbf{c}_{x_{j}}=\mathrm{E}\left[x_{0, j}(m, n) \mathbf{x}_{j+1}(m, n)\right]$ and,

- the cross-correlation vector $\mathbf{c}_{x_{j}} \tilde{y}_{j}=\mathrm{E}\left[y_{j+1}(m, n) \mathbf{x}_{j+1}(m, n)\right]$.

It is important to note that (12) and (21) can be solved if on the one hand, the NSLS decomposition is explicitly defined through the reference prediction and update vectors and, on the other hand, the autocorrelation function $R_{x_{j}}$ and cross-correlation one $R_{x_{j} \tilde{y}_{j}}$ are known at each resolution level $j$. Figures 2 and 3 display the magnitude plots of the frequency responses of the low-pass filter $F_{0}$ for the $5 / 3$ wavelet transform obtained before and after each optimization step applied respectively on a monocular image and a residual one. It is clearly seen that the optimization of the update filter yields an optimal low-pass filter which attenuates better the high frequency components than that obtained without the update optimization step. Furthermore, it is worth pointing out that the optimal low-pass filters corresponding respectively to the texture image and the 
residual one are different since the update filter depends on the contents of the input image to be encoded.

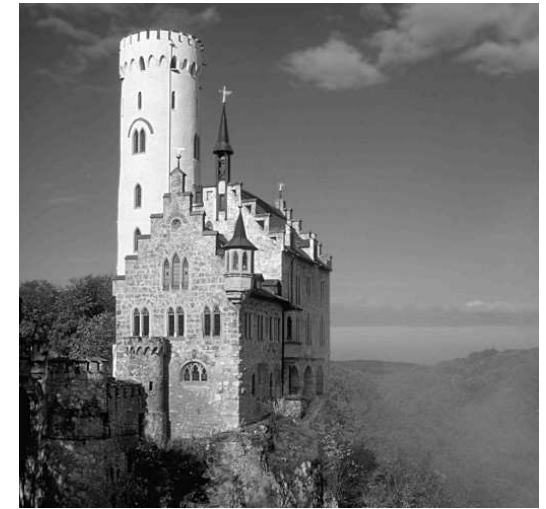

(a): "castle" image

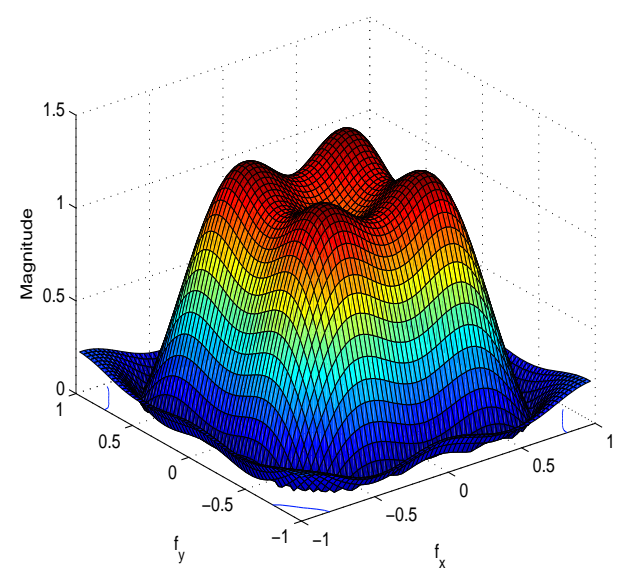

(c)

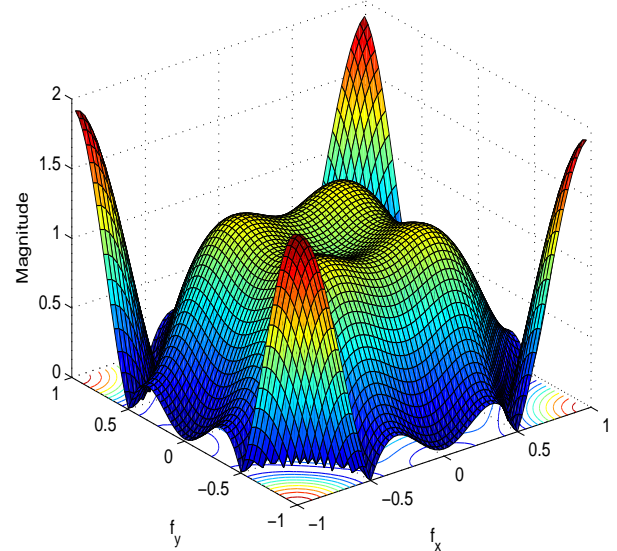

(b)

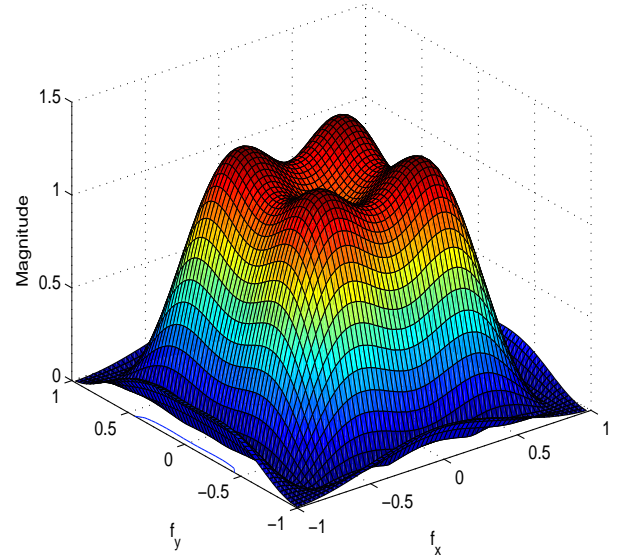

(d)

Figure 2: Frequency responses of the low-pass filter $F_{0}$ when the prediction filters are optimized by minimizing the variance of the detail coefficients, and (b) the update filter is not optimized, (c) the update filter is optimized using Gouze's method (d) the update filter is optimized using the proposed method. 


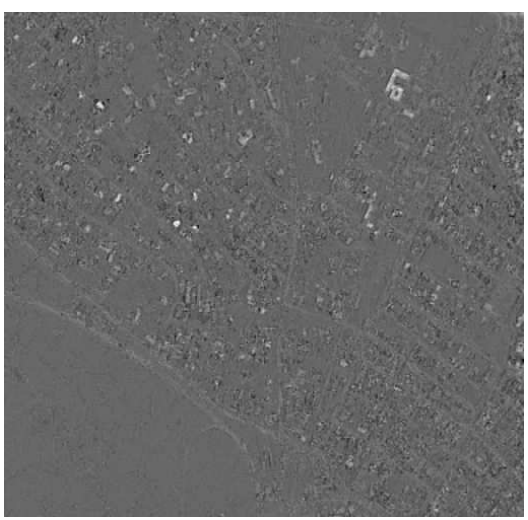

(a): "SPOT5-3" residual image

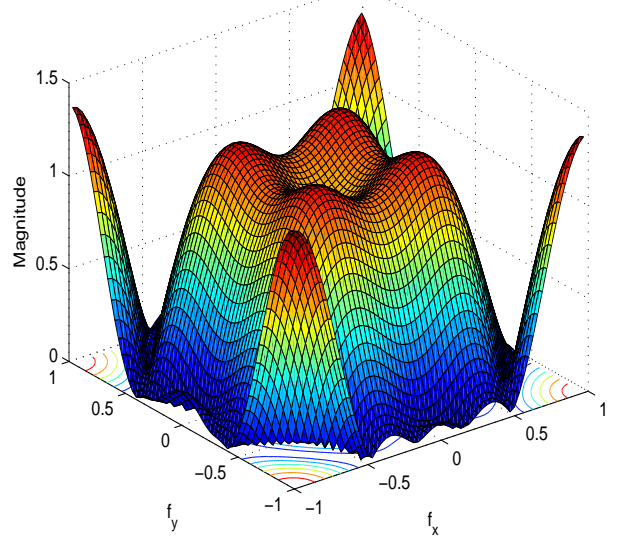

(c)

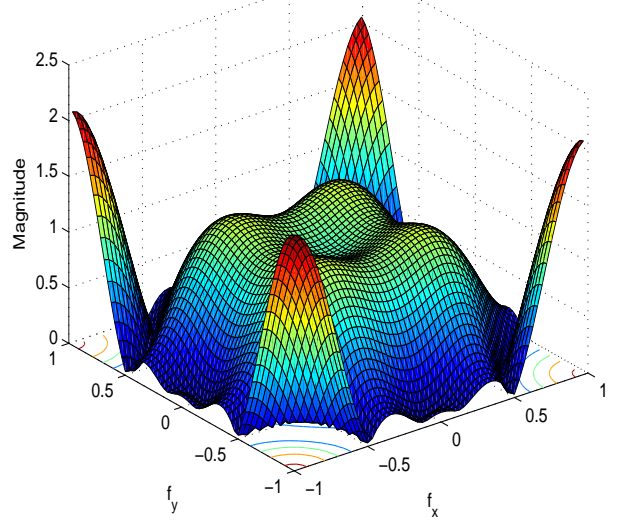

(b)

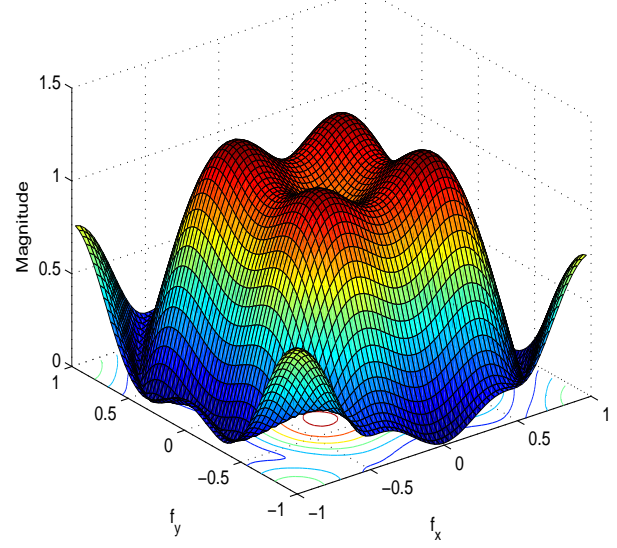

(d)

Figure 3: Frequency responses of the low-pass filter $F_{0}$ when the prediction filters are optimized by minimizing the variance of the detail coefficients, and (b) the update filter is not optimized, (c) the update filter is optimized using Gouze's method (d) the update filter is optimized using the proposed method.

\section{Theoretical analysis}

In this part, we perform a statistical analysis of our method in terms of the adaptation criterion used in the optimization of the update filter. Firstly, we give explicit expressions 
of the optimal update coefficients for a given class of input signals. Then, we confirm that optimizing the update filter leads to lower values of criterion $\mathcal{J}$ than the ones obtained with non-optimized coefficients.

\subsection{Notations}

In order to study the theoretical performance of the optimization method, we will consider the optimization of the 2D non separable $5 / 3$ transform whose underlying operators are given by (7)-(10). This amounts to choosing the following spatial supports for the prediction and update filters:

$$
\begin{aligned}
& \mathcal{P}_{0, j}^{(H H)}=\{(0,0),(0,-1),(-1,0),(-1,-1)\}, \\
& \mathcal{P}_{1, j}^{(H H)}=\{(0,0),(-1,0)\}, \mathcal{P}_{2, j}^{(H H)}=\{(0,0),(0,-1)\}, \\
& \mathcal{P}_{0, j}^{(L H)}=\{(0,0),(-1,0)\}, \mathcal{P}_{1, j}^{(L H)}=\{(0,0),(0,1)\}, \\
& \mathcal{P}_{0, j}^{(H L)}=\{(0,0),(0,-1)\}, \mathcal{P}_{1, j}^{(H L)}=\{(0,0),(1,0)\}, \\
& \mathcal{U}_{j}^{(H L)}=\{(0,0),(0,1)\}, \mathcal{U}_{j}^{(L H)}=\{(0,0),(1,0)\}, \\
& \mathcal{U}_{j}^{(H H)}=\{(0,0),(0,1),(1,0),(1,1)\} .
\end{aligned}
$$

To obtain tractable expressions, we assume that, at a given resolution level $j$, the approximation image $x_{j}$ is modeled as the realization of a first order 2D autoregressive process, the auto-correlation function of which is given by:

$$
\forall(k, l) \in \mathbb{Z}^{2}, \quad R_{x_{j}}(k, l)=\mathrm{E}\left[x_{j}(m, n) x_{j}(m-k, n-l)\right]=\sigma_{j}^{2} \rho_{1, j}^{|k|} \rho_{2, j}^{|l|}
$$

where $\sigma_{j} \in \mathbb{R}_{+}^{*}$, and $\rho_{1, j}$ and $\rho_{2, j} \in[-1,1]$ are the correlation factors along the vertical and horizontal directions.

It is worth noticing that such a model has been widely used to represent a large class of textured images. Furthermore, it is convenient to derive simple theoretical expressions of the filter coefficients. However, the main limitation of this autoregressive model is that it is separable.

\subsection{Optimal prediction coefficients}

Concerning the prediction filter $\mathbf{p}_{j}^{(H H)}$, its optimal coefficients are obtained by solving (12) as described at the beginning of Section 3. Thus, once the autocorrelation 
matrix $\mathrm{E}\left[\tilde{\mathbf{x}}_{j}(m, n) \tilde{\mathbf{x}}_{j}(m, n)^{\top}\right]$ and the cross-correlation vector $\mathrm{E}\left[x_{3, j}(m, n) \tilde{\mathbf{x}}_{j}(m, n)\right]$ are calculated using (27), the optimal weights of $\mathbf{p}_{j}^{(H H)}$ are deduced as follows:

$$
\begin{aligned}
& p_{0, j}^{(H H)}(0,0)=p_{0, j}^{(H H)}(0,-1)=p_{0, j}^{(H H)}(-1,0)=p_{0, j}^{(H H)}(-1,-1)=-\frac{\rho_{1, j} \rho_{2, j}}{\left(1+\rho_{1, j}^{2}\right)\left(1+\rho_{2, j}^{2}\right)} \\
& p_{1, j}^{(H H)}(0,0)=p_{1, j}^{(H H)}(-1,0)=\frac{\rho_{1, j}}{1+\rho_{1, j}^{2}} \\
& p_{2, j}^{(H H)}(0,0)=p_{2, j}^{(H H)}(0,-1)=\frac{\rho_{2, j}}{1+\rho_{2, j}^{2}} .
\end{aligned}
$$

By applying the same optimization method to the other prediction filters $\mathbf{p}_{j}^{(L H)}$ and $\mathbf{p}_{j}^{(H L)}$, we get that their optimal weights are given by:

$$
\begin{aligned}
& p_{0, j}^{(H L)}(0,0)=p_{0, j}^{(H L)}(0,-1)=\frac{\rho_{2, j}}{1+\rho_{2, j}^{2}} \\
& p_{0, j}^{(L H)}(0,0)=p_{0, j}^{(L H)}(-1,0)=\frac{\rho_{1, j}}{1+\rho_{1, j}^{2}} \\
& p_{1, j}^{(H L)}(0,0)=p_{1, j}^{(H L)}(1,0)=p_{1, j}^{(L H)}(0,0)=p_{1, j}^{(L H)}(0,1)=0 .
\end{aligned}
$$

Once the optimal prediction filters are determined, we have to find the optimal weights for the update filter.

\subsection{Optimal update coefficients}

As mentioned above, the optimal update coefficients are optimized by solving (21) where the reference vector $\mathbf{x}_{j+1}(m, n)$ is defined as:

$$
\begin{aligned}
\mathbf{x}_{j+1}(m, n)= & \left(x_{j+1}^{(H L)}(m, n), x_{j+1}^{(H L)}(m, n-1), x_{j+1}^{(L H)}(m, n), x_{j+1}^{(L H)}(m-1, n),\right. \\
& \left.x_{j+1}^{(H H)}(m, n), x_{j+1}^{(H H)}(m, n-1), x_{j+1}^{(H H)}(m-1, n), x_{j+1}^{(H H)}(m-1, n-1)\right)^{\top} .
\end{aligned}
$$

The components of $\boldsymbol{\Gamma}_{j}$ and $\mathbf{c}_{x_{j}}$ can be easily found by using (27). To derive the expression of $\mathbf{c}_{x_{j}} \tilde{y}_{j}$, we have to calculate the cross-correlation between the input signal $x_{j}$ and the output of the ideal low-pass filter $\tilde{y}_{j}$ :

$$
\begin{aligned}
R_{x_{j} \tilde{y}_{j}}(k, l) & =\mathrm{E}\left[\tilde{y}_{j}(m, n) x_{j}(m-k, n-l)\right]=\sum_{p=-\infty}^{p=+\infty} \sum_{q=-\infty}^{q=+\infty} h(p, q) R_{x_{j}}(k-p, l-q) \\
& =\sigma_{j}^{2} \sum_{p=-\infty}^{p=\infty} \sum_{q=-\infty}^{q=+\infty} h(p, q) \rho_{1, j}^{|k-p|} \rho_{2, j}^{|l-q|} .
\end{aligned}
$$


At this point, from (18), we note that $h(p, q)$ has a separable form in $p$ and $q$ :

$$
h(p, q)=h(p) h(q)
$$

where

$$
h(p)=\frac{1}{2} \operatorname{sinc}\left(\frac{p \pi}{2}\right)= \begin{cases}\frac{1}{2} & \text { if } p=0 \\ 0 & \text { if } p \text { is even } \\ \frac{(-1)^{(p-1) / 2}}{p \pi} & \text { if } p \text { is odd. }\end{cases}
$$

As a result, the expression of $R_{x_{j} \tilde{y}_{j}}(k, l)$ can be rewritten in a separable way as:

$$
R_{x_{j} \tilde{y}_{j}}(k, l)=\sigma_{j}^{2} R_{x_{j} \tilde{y}_{j}}^{1}(k) R_{x_{j} \tilde{y}_{j}}^{2}(l)
$$

where

$$
\begin{aligned}
& R_{x_{j} \tilde{y}_{j}}^{1}(k)=\sum_{p=-\infty}^{p=+\infty} h(p) \rho_{1, j}^{|k-p|}, \\
& R_{x_{j} \tilde{y}_{j}}^{2}(l)=\sum_{q=-\infty}^{q=+\infty} h(q) \rho_{2, j}^{|l-q|}
\end{aligned}
$$

In addition, from (35) and (33), we deduce that:

$$
\begin{aligned}
R_{x_{j} \tilde{y}_{j}}^{1}(0)= & \frac{1}{2}+\frac{2}{\pi} \operatorname{arctg}\left(\rho_{1, j}\right), \\
R_{x_{j} \tilde{y}_{j}}^{1}(-1) & =\frac{\rho_{1, j}}{2}+\left(\frac{\rho_{1, j}^{-1}+\rho_{1, j}}{\pi}\right) \operatorname{arctg}\left(\rho_{1, j}\right), \\
R_{x_{j} \tilde{y}_{j}}^{1 D}(2 k)= & \frac{\rho_{1, j}^{2 k}}{2}+\frac{\rho_{1, j}^{2 k}}{\pi}\left(\operatorname{arctg}\left(\rho_{1, j}\right)+\sum_{p=-k}^{-1} \frac{(-1)^{p} \rho_{1, j}^{2 p+1}}{2 p+1}\right) \\
& +\frac{\rho_{1, j}^{-2 k}}{\pi}\left(\operatorname{arctg}\left(\rho_{1, j}\right)-\sum_{p=0}^{k-1} \frac{(-1)^{p} \rho_{1, j}^{2 p+1}}{2 p+1}\right) \text { if } k>0, \\
R_{x_{j} \tilde{y}_{j}}^{1}(2 k)= & \frac{\rho_{1, j}^{-2 k}}{2}+\frac{\rho_{1, j}^{2 k}}{\pi}\left(\operatorname{arctg}\left(\rho_{1, j}\right)-\sum_{p=0}^{-k-1} \frac{(-1)^{p} \rho_{1, j}^{2 p+1}}{2 p+1}\right) \\
& +\frac{\rho_{1, j}^{-2 k}}{\pi}\left(\operatorname{arctg}\left(\rho_{1, j}\right)+\sum_{p=k}^{-1} \frac{(-1)^{p} \rho_{1, j}^{2 p+1}}{2 p+1}\right) \quad \text { if } k<0
\end{aligned}
$$




$$
\begin{array}{r}
R_{x_{j} \tilde{y}_{j}}^{1}(2 k+1)=\frac{\rho_{1, j}^{2 k+1}}{2}+\frac{\rho_{1, j}^{2 k+1}}{\pi}\left(\operatorname{arctg}\left(\rho_{1, j}\right)+\sum_{p=-k-1}^{-1} \frac{(-1)^{p} \rho_{1, j}^{2 p+1}}{2 p+1}\right) \\
+\frac{\rho_{1, j}^{-(2 k+1)}}{\pi}\left(\operatorname{arctg}\left(\rho_{1, j}\right)-\sum_{p=0}^{-k} \frac{(-1)^{p} \rho_{1, j}^{2 p+1}}{2 p+1}\right) \text { if } k>-1, \\
R_{x_{j} \tilde{y}_{j}}^{1}(2 k+1)=\frac{\rho_{1, j}^{-(2 k+1)}}{2}+\frac{\rho_{1, j}^{2 k+1}}{\pi}\left(\operatorname{arctg}\left(\rho_{1, j}\right)-\sum_{p=0}^{-k-2} \frac{(-1)^{p} \rho_{1, j}^{2 p+1}}{2 p+1}\right) \\
+\frac{\rho_{j}^{-(2 k+1)}}{\pi}\left(\operatorname{arctg}\left(\rho_{1, j}\right)+\sum_{p=k+1}^{-1} \frac{(-1)^{p} \rho_{1, j}^{2 p+1}}{2 p+1}\right) \text { if } k<-1 .
\end{array}
$$

The expressions of $R_{x_{j} \tilde{y}_{j}}^{2}(l)$ are easily derived by replacing $\rho_{1, j}$ by $\rho_{2, j}$ in the above equalities. Altogether, we obtain $R_{x_{j} \tilde{y}_{j}}(k, l)$ by using (34).

Once $\boldsymbol{\Gamma}_{j}, \mathbf{c}_{x_{j}}$ and $\mathbf{c}_{x_{j} \tilde{y}_{j}}$ are determined, after some calculations, the optimal update coefficients are simply given by:

$$
\begin{aligned}
& u_{j}^{(H L)}(0,0)=u_{j}^{(H L)}(0,1)=\frac{\pi+4 \operatorname{arctg}\left(\rho_{1, j}\right)}{2 \pi^{2}} \\
& u_{j}^{(L H)}(0,0)=u_{j}^{(L H)}(1,0)=\frac{\pi+4 \operatorname{arctg}\left(\rho_{2, j}\right)}{2 \pi^{2}} \\
& u_{j}^{(H H)}(0,0)=u_{j}^{(H H)}(0,1)=u_{j}^{(H H)}(1,0)=u_{j}^{(H H)}(1,1)=\frac{1}{\pi^{2}} .
\end{aligned}
$$

Note that, although the simple separable autoregressive modeling which was adopted induces a separable form of the optimized prediction and update filters, the resulting NSLS cannot be put under the classical form of a separable lifting structure. Indeed, the vector $\mathbf{u}_{j}^{(H H)}$ is not equal to the Kronecker product of $\mathbf{u}_{j}^{(H L)}$ and $\mathbf{u}_{j}^{(L H)}$. The necessary conditions for satisfying the equivalence between a non separable scheme and a separable one can be found in [17].

\subsection{Adaptation criterion values}

In order to show the interest of our optimization approach, we aim at comparing the values of the criterion $\mathcal{J}$ in the two following cases:

- Case I: the update vector $\mathbf{u}_{j}^{\text {nonopt }}$ has non optimized weights corresponding to the conventional $5 / 3$ transform, given in (10): $\mathbf{u}_{j}^{\text {nonopt }}=[1 / 4,1 / 4,1 / 4,1 / 4,-1 / 16,-1 / 16,-1 / 16,-1 / 16]$.

- Case II: the update vector $\mathbf{u}_{j}^{\text {opt }}$ corresponds to weights minimizing $\mathcal{J}$. 
Our objective is to evaluate the gain resulting from the optimization of $\mathcal{J}$ by calculating $\mathcal{J}\left(\mathbf{u}_{j}^{\text {nonopt }}\right)$ and $\mathcal{J}\left(\mathbf{u}_{j}^{\text {opt }}\right)$. To this respect, we recall that the optimal value of $\mathcal{J}$ can be expressed as:

$$
\begin{aligned}
\mathcal{J}\left(\mathbf{u}_{j}^{\mathrm{opt}}\right)= & \mathrm{E}\left[x_{0, j}(m, n)^{2}\right]+\mathrm{E}\left[y_{j+1}(m, n)^{2}\right]-2 \mathrm{E}\left[x_{0, j}(m, n) y_{j+1}(m, n)\right] \\
& +\left(\mathbf{u}_{j}^{\mathrm{opt}}\right)^{\top} \mathrm{E}\left[x_{0, j}(m, n) \mathbf{x}_{j+1}(m, n)\right]-\left(\mathbf{u}_{j}^{\mathrm{opt}}\right)^{\top} \mathrm{E}\left[y_{j+1}(m, n) \mathbf{x}_{j+1}(m, n)\right]
\end{aligned}
$$

Eq. (39) indicates that the evaluation of $\mathcal{J}$ requires to find the expressions of $R_{x_{j}}(k, l)$, $R_{x_{j} \tilde{y}_{j}}(k, l)$, and the value $R_{y_{j+1}}(0,0)$ of the autocorrelation of $y_{j+1}$ at $(0,0)$. The first two expressions are readily given by (27) and (37) whereas the third one is easily derived:

$$
R_{y_{j+1}}(0,0)=\sigma_{j}^{2}\left(\frac{1}{2}+\frac{2}{\pi} \operatorname{arctg}\left(\rho_{1, j}\right)\right)^{2}\left(\frac{1}{2}+\frac{2}{\pi} \operatorname{arctg}\left(\rho_{2, j}\right)\right)^{2}
$$

It is worth pointing out that the calculation of the adaptation criterion has not led to a simple expression as a function of $\rho_{1, j}$ and $\rho_{2, j}$. In order to obtain more easily interpretable results, we propose to consider the case of an approximation image that presents strong correlations in the horizontal and vertical directions. In other words, we focus on the case when the couple $\left(\rho_{1, j}, \rho_{2, j}\right)$ is close to $(1,1)$. Hence, we propose to approximate $\mathcal{J}$ by its second-order Taylor expansion $\widehat{\mathcal{J}}$ when $\left(\rho_{1, j}, \rho_{2, j}\right)$ is around $(1,1)$ :

$$
\begin{aligned}
\widehat{\mathcal{J}}\left(\mathbf{u}_{j}^{\text {nonopt }}\right)= & \frac{\sigma_{j}^{2}}{2}\left[\frac{1}{4}\left(2-\rho_{1, j}-\rho_{2, j}\right)+\frac{1}{8}\left(1-\rho_{1, j}\right)^{2}+\frac{1}{8}\left(1-\rho_{2, j}\right)^{2}\right. \\
& \left.+\left(\frac{3}{\pi^{2}}+\frac{1}{32}\right)\left(1-\rho_{1, j}\right)\left(1-\rho_{2, j}\right)\right] \\
\widehat{\mathcal{J}}\left(\mathbf{u}_{j}^{\text {opt }}\right)= & \frac{\sigma_{j}^{2}}{\pi^{2}}\left[(\pi-2)\left(2-\rho_{1, j}-\rho_{2, j}\right)+\frac{\pi-2}{2}\left(1-\rho_{1, j}\right)^{2}\right. \\
+ & \left.\frac{\pi-2}{2}\left(1-\rho_{2, j}\right)^{2}+\frac{\pi^{2}-8 \pi+4}{\pi^{2}}\left(1-\rho_{1, j}\right)\left(1-\rho_{2, j}\right)\right] .
\end{aligned}
$$

\subsection{Discussion}

In order to emphasize the usefulness of the optimization of the update filter, we illustrate in Fig. 4 the variations of the criterion in $(41)$ with respect to $\rho_{j}$ where we have assumed that $\rho_{1, j}=\rho_{2, j}=\rho_{j}$. At first, Fig. 4 shows that the Taylor expansion closely approximates the criterion when $\rho_{j} \geq 0.85$.

Note that in practice, for natural images, values of $\rho_{j}$ are within such a range [35].

Besides, by adapting the update filter coefficients to the image statistical characteristics, 


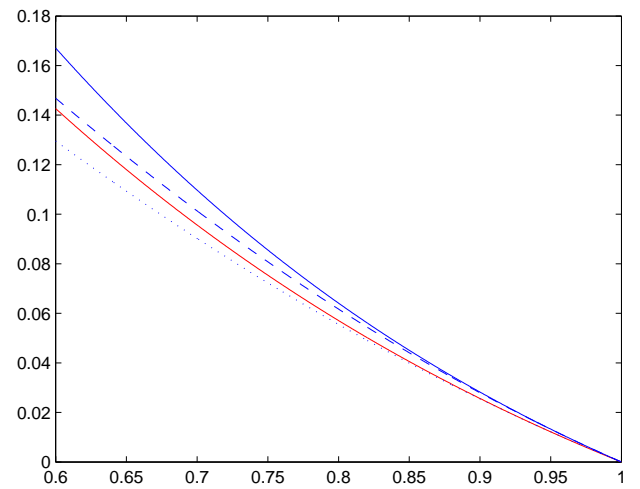

Figure 4: Variations of the adaptation criterion w.r.t $\rho_{j}: \mathcal{J}\left(\mathbf{u}^{\text {nonopt }}\right)$ in solid blue line, $\mathcal{J}\left(\mathbf{u}^{\text {opt }}\right)$ in solid red line, $\widehat{\mathcal{J}}\left(\mathbf{u}^{\text {nonopt }}\right)$ in dotted blue line, $\widehat{\mathcal{J}}\left(\mathbf{u}^{\text {opt }}\right)$ in dotted red line.

we can see that the optimized scheme leads to an improvement compared with the nonoptimized one. In summary, the interest of this theoretical study is twofold. First, it allows us to better understand the benefit that can be drawn from the proposed optimization method. Furthermore, it provides closed form expressions of the prediction and update filters which can be used in order to simplify the implementation of the proposed optimization method.

\section{Coding techniques}

\subsection{Coding of the wavelet coefficients}

After applying our decomposition, the generated coefficients must be encoded. However, the coding scheme should enable quality scalability for progressive reconstruction purposes. This is basically achieved by sending the coefficients in decreasing order of their importance. In other words, the most significant ones are first encoded at a reduced accuracy. So, a first approximation image is produced, which is further gradually refined by decoding the less significant coefficients. To this end, several scalable codecs have been developed $[36,37,38,39]$. The main advantage of these embedded codecs is that the encoder can terminate the encoding at any point, thereby allowing a target bitrate to be met exactly. Similarly, the decoder can also stop decoding at any point resulting in the image that would have been produced at the rate corresponding to the truncated bitstream. Among these various codecs, it is shown in [39] that EZBC and 
JPEG 2000 codecs are the most effective in terms of compression and reconstruction quality. In this paper, we have chosen the JPEG 2000 codec [38] which yields excellent performances in terms of compression efficiency and quality of reconstruction.

\subsection{Transmission cost of the filter coefficients}

The entire set of optimal weights for the update and the three prediction filters corresponds to an amount of $o_{p, u}=\left(L_{p}+L_{u}\right) J$ floating point coefficients, where $L_{p}$ and $L_{u}$ denote respectively the number of prediction and update weights in the adaptive NSLS and $J$ represents the number of resolution levels. For an image of size $N_{1} \times N_{2}$, the transmission cost of the filters coefficients will increase the bitrate achieved by the proposed optimization method, by $\frac{32 o_{p, u}}{N_{1} N_{2}}$ bits per pixel. For example, if we consider the supports of the prediction and update filters corresponding to the 2D 5/3 transform with $L_{p}=16$ and $L_{u}=8$ (see (7)-(10)), when $N_{1}=N_{2}=512, J=2$ and if the weights are stored on 32 bits, the bitrate will be increased by 0.0059 bpp which is a very small fraction of the overall data bitrate. However, if we use the explicit expressions of the filter coefficients given by the theoretical analysis, it is enough to send only the correlation factors $\rho_{1, j}$ and $\rho_{2, j}$. This leads to a significant reduction of the transmission cost of the filter coefficients, which becomes $0.0004 \mathrm{bpp}$.

\section{Experimental results}

Simulations were carried out on two kinds of still images originally quantized on 8 bpp which are either monocular single views ${ }^{2}$ or stereoscopic ones ${ }^{3}$. The gain related to the optimization of the NSLS operators was evaluated in these contexts.

Since we are mainly interested in lossy-to-lossless coding schemes, we will consider the integer-to-integer $5 / 3$ transform recommended for the lossless mode of JPEG2000 [4]. In order to show the benefits of the optimization of all the involved operators, we provide the results for the following decompositions carried out over two resolution levels:

- The first one is the lifting scheme corresponding to the $5 / 3$ transform, which is known as the $(2,2)$ integer wavelet transform [7]. Its underlying decomposition operators are

\footnotetext{
${ }^{2}$ http://sipi.usc.edu/database

${ }^{3}$ http://vasc.ri.cmu.edu/idb/html/stereo/index.html
} 
given by (7)-(10). In the following, this method will be designated by NSLS(2,2).

- The second variant consists of optimizing only the prediction filters of the NSLS(2,2) while the update filter is kept unchanged. Note that the prediction support is preserved. This method will be denoted by NSLS(2,2)-OPT1.

- The third method consists of optimizing both the prediction and update operators. In what follows, our proposed method will be designated by NSLS(2,2)-OPT2-PM1 (resp. NSLS(2,2)-OPT2-PM2) when using the experimental (resp. theoretical) optimal update coefficients.

- We have also tested for comparison the update optimization method of Gouze et al. which aims at designing the update filter in order to minimize a reconstruction error [30, 31]. This optimization method will be designated by NSLS(2,2)-OPT2-GM.

As mentioned earlier, the proposed method for the design of all filters can be applied to any classical P-U lifting structure. To illustrate the performance of the optimization method when using longer filters, we will consider a simple example corresponding to the $(4,2)$ integer wavelet transform. Indeed, after applying this transform to the lines then to the columns, it is easy to show that the corresponding support of the two-dimensional prediction and update filters are given by:

$$
\begin{aligned}
& \mathcal{P}_{0, j}^{(H H)}=\left\{(k, l)_{-2 \leq k \leq 1,-2 \leq l \leq 1}\right\}, \mathcal{P}_{1, j}^{(H H)}=\left\{(k, 0)_{-2 \leq k \leq 1}\right\}, \mathcal{P}_{2, j}^{(H H)}=\left\{(0, l)_{-2 \leq l \leq 1}\right\}, \\
& \mathcal{P}_{0, j}^{(L H)}=\left\{(k, l)_{-2 \leq k \leq 1,-2 \leq l \leq 1}\right\}, \mathcal{P}_{1, j}^{(L H)}=\{(0,0),(0,1)\}, \\
& \mathcal{P}_{0, j}^{(H L)}=\left\{(k, l)_{-2 \leq k \leq 1,-2 \leq l \leq 1}\right\}, \mathcal{P}_{1, j}^{(H L)}=\{(0,0),(1,0)\}, \\
& \mathcal{U}_{j}^{(0)}=\{(0,0),(0,1)\}, \mathcal{U}_{j}^{(1)}=\{(0,0),(1,0)\}, \mathcal{U}_{j}^{(2)}=\{(0,0),(0,1),(1,0),(1,1)\}
\end{aligned}
$$

In what follows, NSLS(4,2)-OPT1 and NSLS(4,2)-OPT2-PM1 will designate respectively the decompositions before and after the optimization of the update filter.

First of all, the performance of the proposed method has been assessed on monocular images. Starting with the evaluation of these decompositions in a progressive reconstruction context, Figures 5 and 6 give the variations of the PSNR versus the bitrate for the images "lena" and "castle". 


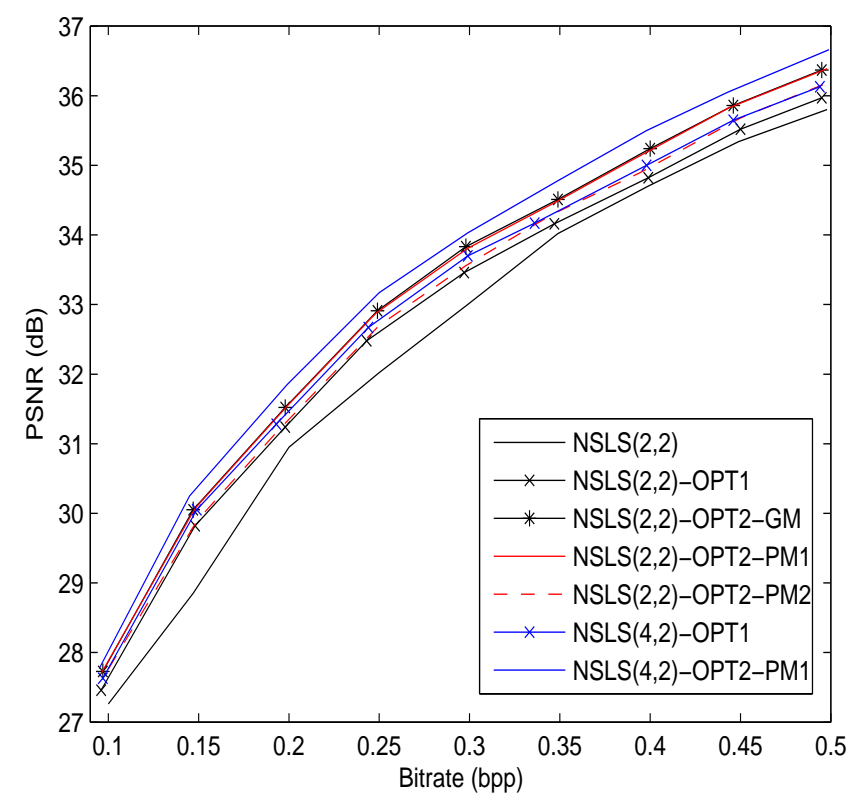

Figure 5: PSNR (in dB) versus the bitrate (bpp) after JPEG2000 progressive encoding for the "lena" image.

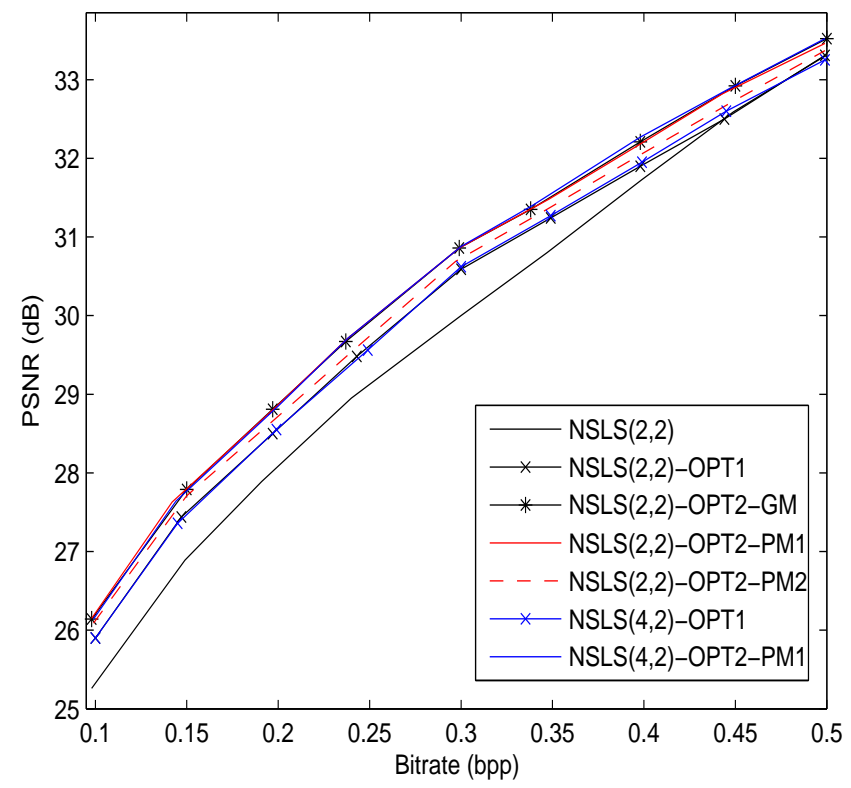

Figure 6: PSNR (in dB) versus the bitrate (bpp) after JPEG2000 progressive encoding for the "castle" image. 
It is worth pointing out that NSLS(2,2)-OPT2-PM1 outperforms NSLS(2,2) by 0.2$0.9 \mathrm{~dB}$ and NSLS(2,2)-OPT1 by about $0.4 \mathrm{~dB}$. It can be noticed that the proposed update optimization method NSLS(2,2)-OPT2-PM1 and Gouze's method NSLS(2,2)OPT2-GM have similar performances in terms of quality of reconstruction. However, it is worth pointing out that our work present two advantages with respect to Gouze's work. Firstly, the proposed method reduces significantly the complexity of the optimization algorithm. Indeed, although both minimization approaches amount to solving a linear system $\mathbf{A}_{j} \mathbf{u}_{j}=\mathbf{b}_{j}$, it is worth noting that calculating $\mathbf{A}_{j}$ and $\mathbf{b}_{j}$ is more intricate and requires more operations in the case of Gouze's method [30, 31]. More precisely, in this case, the matrix $\mathbf{A}_{j}$ (resp. vector $\mathbf{b}_{j}$ ) is completely defined by the computation of four matrices of size $8 \times 8$ (resp. four vectors of dimension 8 ) since the reconstruction error is evaluated for each of the four polyphase components (see (13)). Our optimization method requires only the computation of one matrix of size $8 \times 8$ and two vectors of dimension 8 as shown in (21). To better illustrate this fact, we propose to compare these two optimization methods in terms of computation time.

Table 1: Computation time of the optimization method of the update filter (in seconds).

\begin{tabular}{|l|l|l|l|l|}
\hline Image & lena & einst & castle & elaine \\
\hline Gouze's method & 3.14 & 3.11 & 3.09 & 3.11 \\
\hline Our method & 1.06 & 1.07 & 1.08 & 1.06 \\
\hline
\end{tabular}

Table 1 presents the computation time of a Matlab implementation of the optimization methods of the update filter for some images of size $512 \times 512$. Simulations are carried out by using an Intel Core $2(3 \mathrm{GHz})$ computer. We can note that the proposed method is three times faster than Gouze's method based on the minimization of the reconstruction error. Secondly, the main advantage of the current work is that it provides explicit expressions of the optimal filter coefficients based only on the horizontal and vertical correlation factors. In order to show the benefit of the statistical analysis we conducted, we evaluate our method when using directly the obtained theoretical filter coefficient expressions. We can see in Figures 5 and 6 that using the theoretical values of the optimal filters NSLS(2,2)-OPT2-PM2 yields results comparable with the method based on experimental filter coefficients NSLS(2,2)-OPT2-PM1. This confirms that the 
considered autoregressive model allows us to obtain theoretical results which are close to the experimental ones. We however noticed that the simple autoregressive model becomes inaccurate in the case of some specific images showing a strong correlation in another direction than the horizontal or vertical ones. For example, Table 2 illustrates the optimized filter coefficients for the "castle" and "straw" images. An additional gain of $0.3 \mathrm{~dB}$ can be achieved by using longer filters NSLS(4,2)-OPT2-PM1.

Table 2: Experimental and theoretical results of filter coefficients for the "castle" and "straw" images

\begin{tabular}{|c|l|l|l|}
\hline Image & Filter & Experimental optimized coefficients & Theoretical optimized coefficients \\
\hline \multirow{5}{*}{ castle } & $\mathbf{p}_{0}^{(H H)}$ & {$[-0.2153,-0.2041,-0.2081,-0.2139$,} & {$[-0.2496,-0.2496,-0.2496,-0.2496$,} \\
& & $0.4749,0.4798,0.4457,0.4419]^{\top}$ & $0.4997,0.4997,0.4997,0.4997]^{\top}$ \\
\cline { 2 - 5 } & $\mathbf{p}_{0}^{(H L)}$ & {$[0.5041,0.5054,-0.1192,-0.1081]^{\top}$} & {$[0.4994,0.4994,0,0]^{\top}$} \\
\cline { 2 - 5 } & $\mathbf{p}_{0}^{(L H)}$ & {$[0.4983,0.5039,-0.1494,-0.1542]^{\top}$} & {$[0.4997,0.4997,0,0]^{\top}$} \\
\cline { 2 - 5 } & $\mathbf{u}_{0}$ & {$[0.2897,0.2855,0.2839,0.2771$,} & {$[0.3150,0.3150,0.3132,0.3132$,} \\
& & $0.0906,0.0753,0.0827,0.0849]^{\top}$ & $0.1013,0.1013,0.1013,0.1013]^{\top}$ \\
\hline \hline \multirow{3}{*}{ straw } & $\mathbf{p}_{0}^{(H H)}$ & {$[-0.2410,0.0855,0.0676,-0.2505$,} & {$[-0.2293,-0.2293,-0.2293,-0.2293$,} \\
& & $0.3446,0.3360,0.3160,0.3289]^{\top}$ & $0.4769,0.4769,0.4808,0.4808]^{\top}$ \\
\cline { 2 - 5 } & $\mathbf{p}_{0}^{(H L)}$ & {$[0.5206,0.5298,-0.0763,-0.0659]^{\top}$} & {$[0.4808,0.4808,0,0]^{\top}$} \\
\cline { 2 - 5 } & $\mathbf{p}_{0}^{(L H)}$ & {$[0.5568,0.4904,-0.1051,-0.1554]^{\top}$} & {$[0.4769,0.4769,0,0]^{\top}$} \\
\cline { 2 - 5 } & $\mathbf{u}_{0}$ & {$[0.1990,0.1881,0.1744,0.1949$,} & {$[0.2874,0.2874,0.2901,0.2901$,} \\
& & $0.2118,-0.0928,-0.0949,0.2110]^{\top}$ & $0.1013,0.1013,0.1013,0.1013]^{\top}$ \\
\hline
\end{tabular}

Figures 7, 8 and 9 display the reconstructed images of "airport" and "lena" and "elaine".

The quality of these images is compared both in terms of PSNR and Structural SIMilarity (SSIM) [40]. It can be observed that optimizing both the prediction and update filters significantly improves the quality of reconstruction. The difference in PSNR (resp. SSIM) ranges from $0.35 \mathrm{~dB}$ to $0.4 \mathrm{~dB}$ (resp. from 0.03 to 0.07 ) compared with the decompositions in which only the prediction filters are optimized. In addition, we have tested these methods in a lossless coding context and the related final bitrates are given in Table 3. Slight improvements (about 0.02 bpp compared to the Gouze's method) are obtained when our optimization method is employed in a lossless coding scheme. 


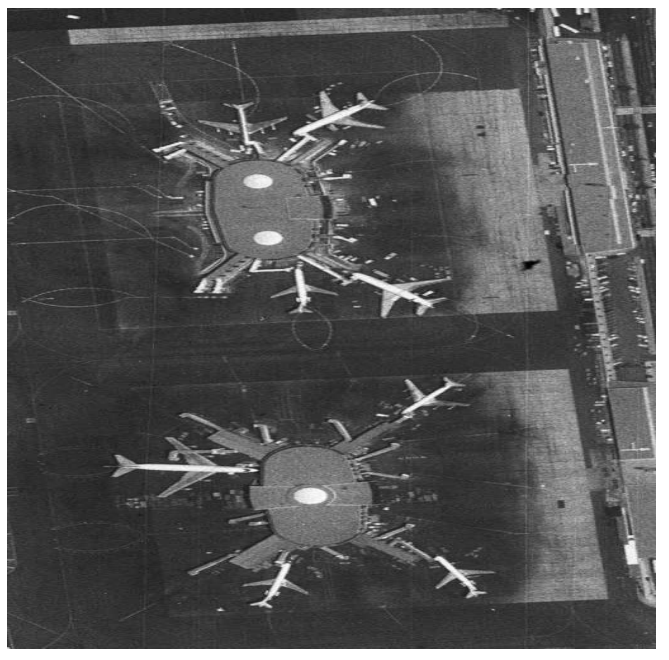

(a): Original image

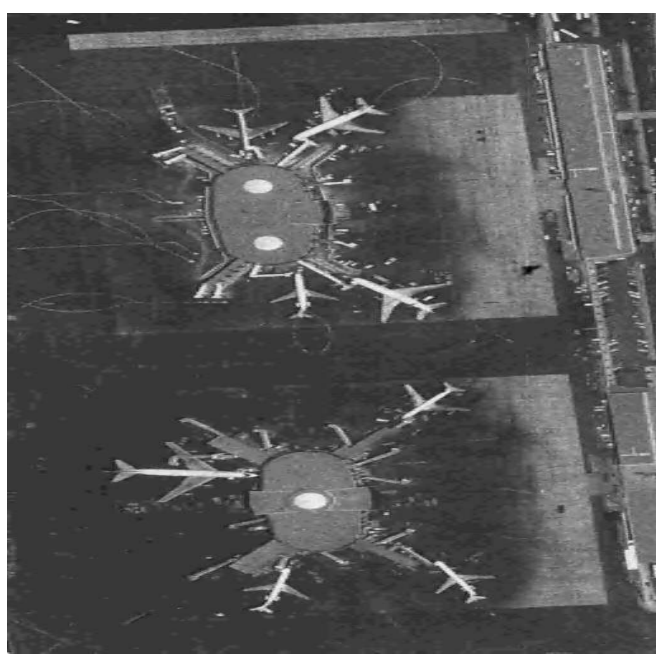

(c): $\mathrm{PSNR}=28.45 \mathrm{~dB}, \mathrm{SSIM}=0.738$

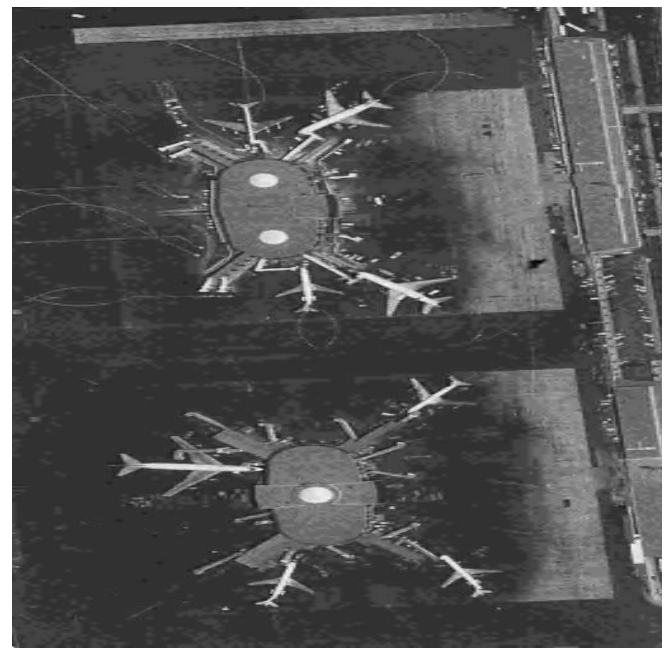

(b): $\mathrm{PSNR}=28.42 \mathrm{~dB}, \mathrm{SSIM}=0.734$

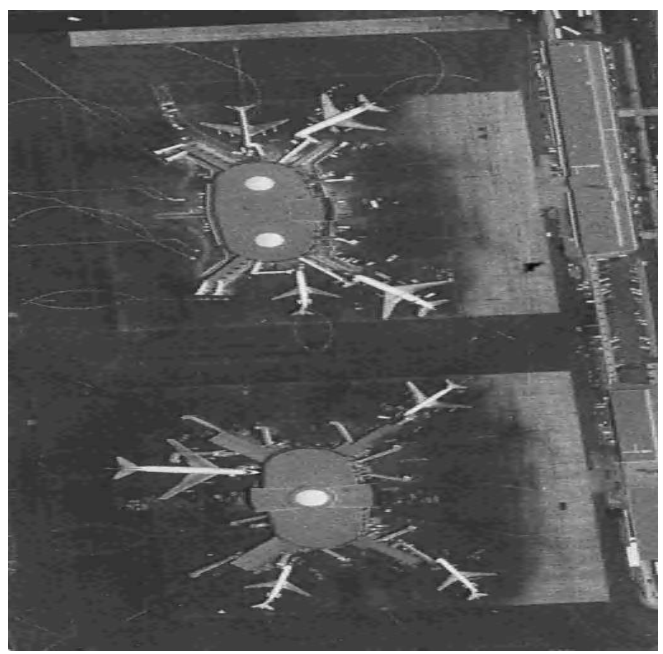

(d): PSNR=28.80 dB, SSIM=0.741

Figure 7: Zoom applied on the reconstructed "airport" image at 0.35 bpp using: (b) NSLS(2,2) (c) $\operatorname{NSLS}(2,2)-O P T 1$ (d) NSLS(2,2)-OPT2-PM1. 


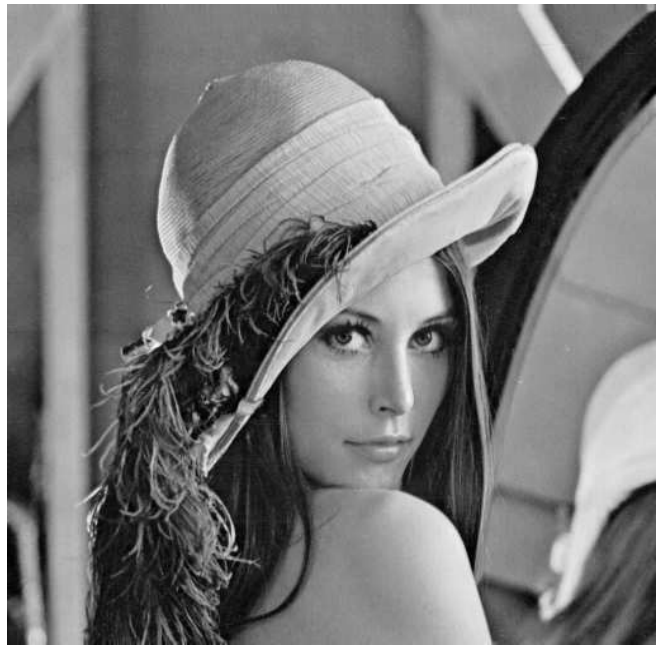

(a): Original image

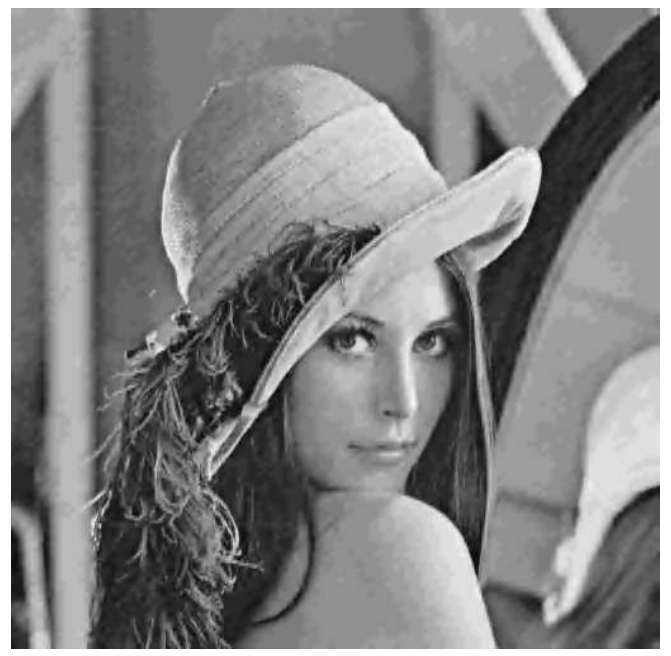

(c): $\mathrm{PSNR}=32.49 \mathrm{~dB}, \mathrm{SSIM}=0.872$

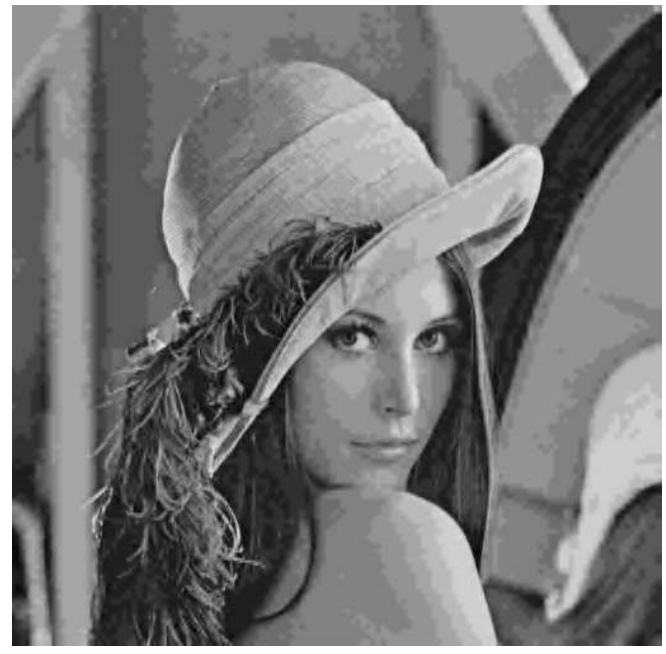

(b): $\mathrm{PSNR}=31.81 \mathrm{~dB}, \mathrm{SSIM}=0.860$

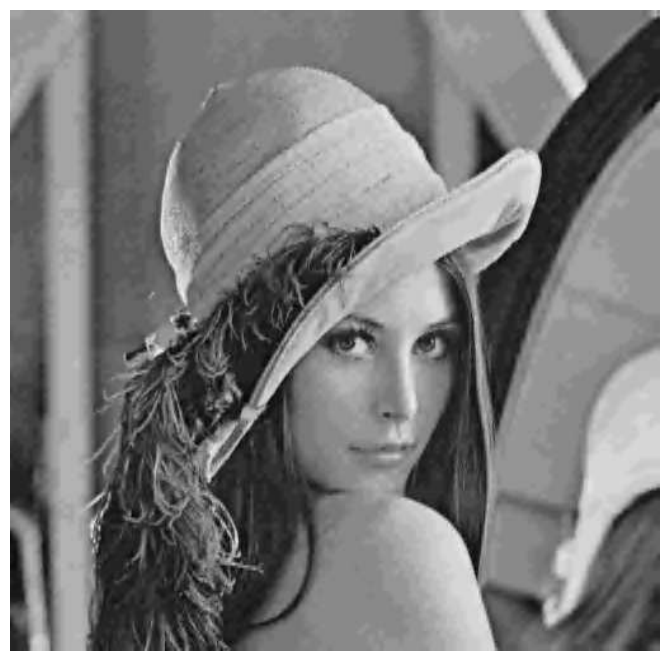

(d): PSNR $=32.92 \mathrm{~dB}, \mathrm{SSIM}=0.877$

Figure 8: The reconstructed "lena" image at 0.25 bpp using: (b) NSLS(2,2) (c) NSLS(2,2)-OPT1 (d) $\operatorname{NSLS}(2,2)-O P T 2-P M 1$. 


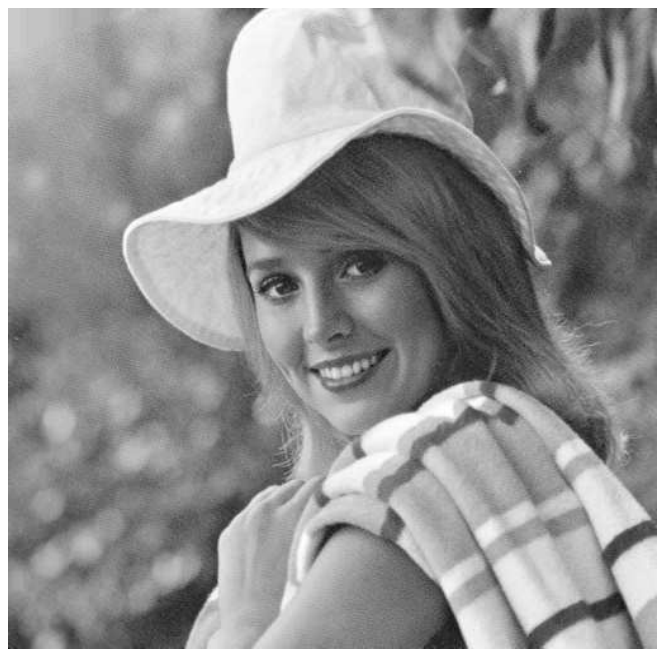

(a): Original image

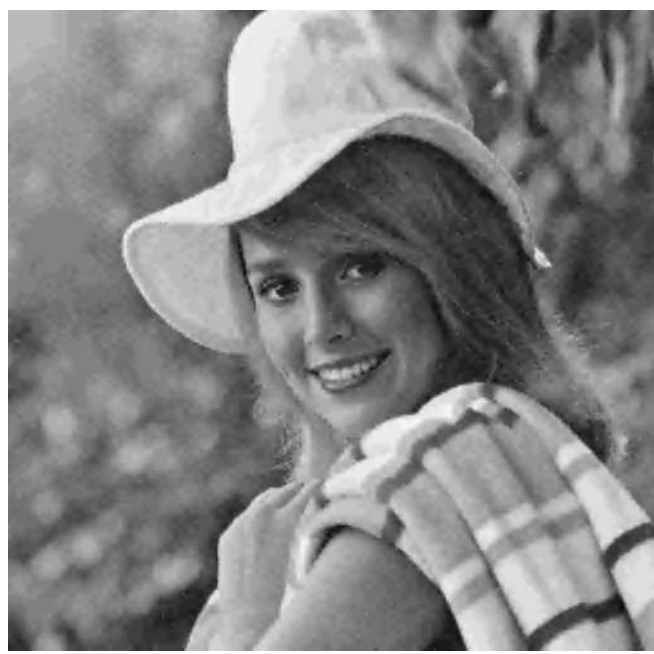

(c): PSNR $=30.99 \mathrm{~dB}, \mathrm{SSIM}=0.741$

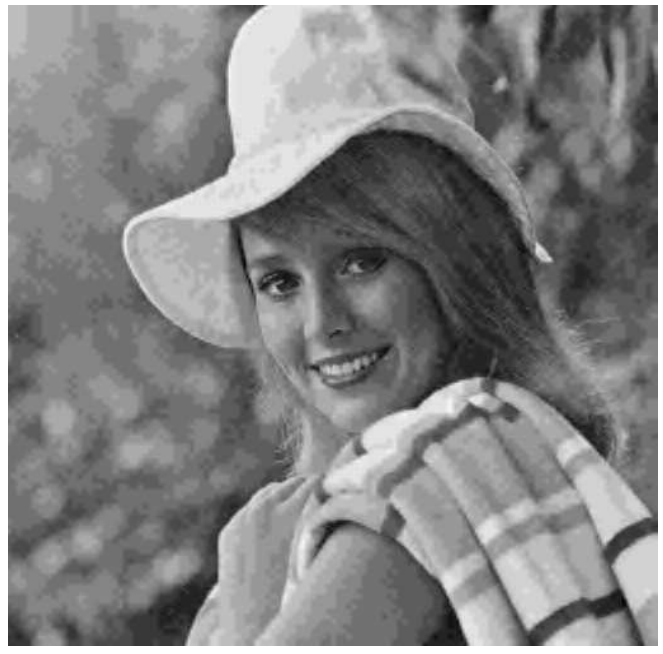

(b): $\mathrm{PSNR}=30.64 \mathrm{~dB}, \mathrm{SSIM}=0.733$

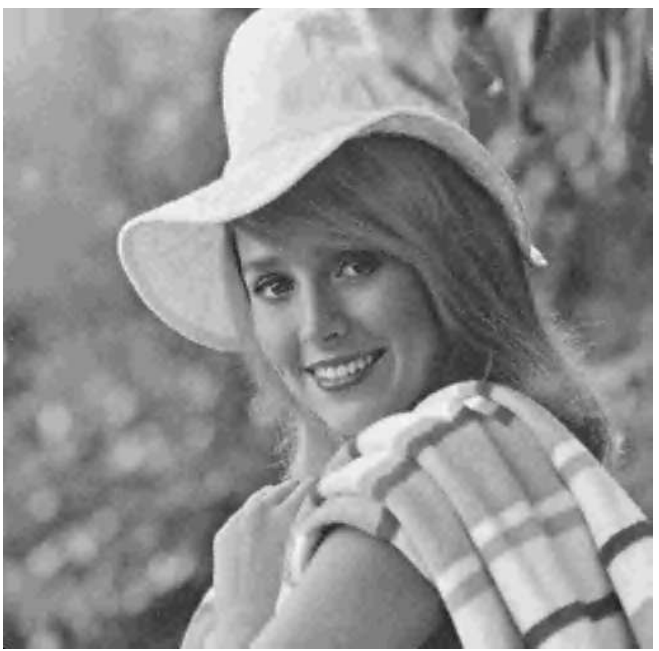

(d): PSNR $=31.37 \mathrm{~dB}, \mathrm{SSIM}=0.748$

Figure 9: The reconstructed "elaine" image at 0.2 bpp using: (b) NSLS(2,2) (c) NSLS(2,2)-OPT1 (d) $\operatorname{NSLS}(2,2)-O P T 2-P M 1$. 
Table 3: Performance of the lossless decompositions in terms of bitrate (bpp) using JPEG2000.

\begin{tabular}{|l|l|l|l|l|}
\hline Image & NSLS $(2,2)$ & $\begin{array}{l}\text { NSLS(2,2) } \\
\text { OPT1 }\end{array}$ & $\begin{array}{l}\text { NSLS(2,2)- } \\
\text { OPT2-GM }\end{array}$ & $\begin{array}{l}\text { NSLS(2,2)- } \\
\text { OPT2-PM1 }\end{array}$ \\
\hline spot5-1 & 3.94 & 3.87 & 3.88 & 3.85 \\
\hline spot5-2 & 4.17 & 4.09 & 4.09 & 4.06 \\
\hline spot5-3 & 4.15 & 4.07 & 4.08 & 4.04 \\
\hline lena & 4.31 & 4.31 & 4.31 & 4.31 \\
\hline straw & 6.35 & 6.34 & 6.34 & 6.34 \\
\hline airport & 5.49 & 5.48 & 5.48 & 5.48 \\
\hline earthquake & 6.48 & 6.30 & 6.33 & 6.30 \\
\hline castle & 3.70 & 3.66 & 3.66 & 3.66 \\
\hline \hline Average & 4.82 & 4.76 & 4.77 & 4.75 \\
\hline
\end{tabular}

The second part of the experiments is concerned with stereo images. More precisely, we also evaluate our optimization method for coding residual image obtained after disparity-compensation between the right and the left views. Figures 10 and 11 illustrate the evolution of the PSNR versus the bitrate of the residual image generated respectively from the "shrub" and "spot5-3" stereo image. An improvement of 0.1-0.25 dB is obtained by optimizing the update filter. The gain becomes more important (up to $0.6 \mathrm{~dB}$ ) when compared with the non-optimized $\operatorname{NSLS}(2,2)$ wavelet transform. Furthermore, we show that using the theoretical filter coefficient expressions, NSLS(2,2)-OPT2-PM2 yields performances similar to the NSLS(2,2)-OPT2-PM1 scheme. An additional gain of $0.3 \mathrm{~dB}$ is achieved by using NSLS(4,2)-OPT2-PM1. Figure 12 displays the reconstructed images of the residual one generated from the "pentagone" stereo pair. It can be observed that optimizing both the prediction and update filters leads to an improvement of $0.22 \mathrm{~dB}$ (resp. 0.04) in PSNR (resp. SSIM) compared with the decomposition in which only the prediction filters are optimized. 


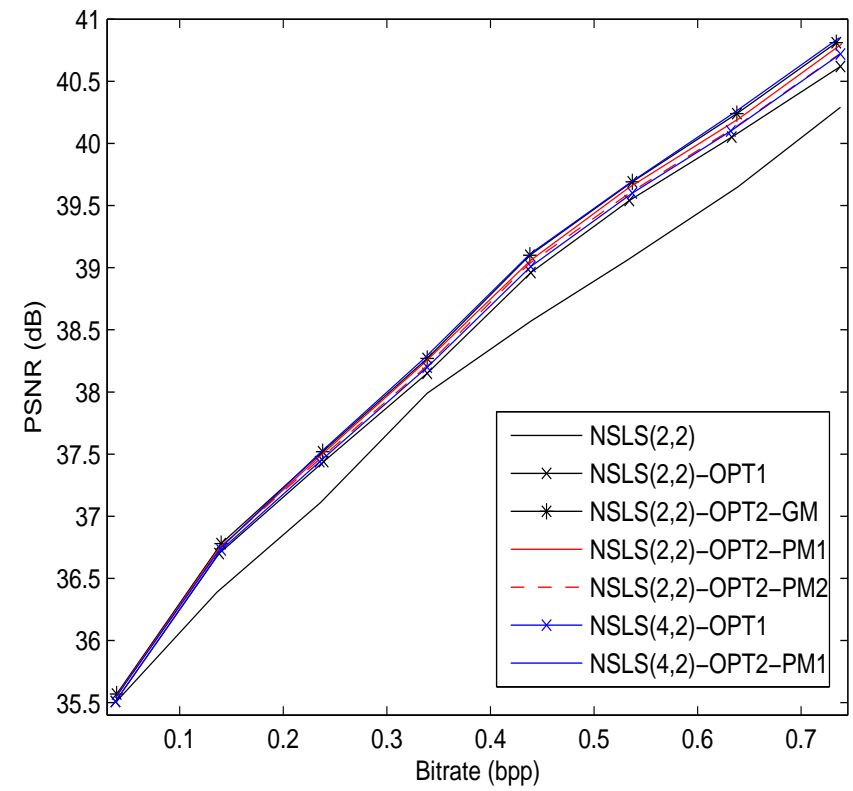

Figure 10: PSNR (in dB) versus the bitrate (bpp) after JPEG2000 progressive encoding for the "shrub" residual image.

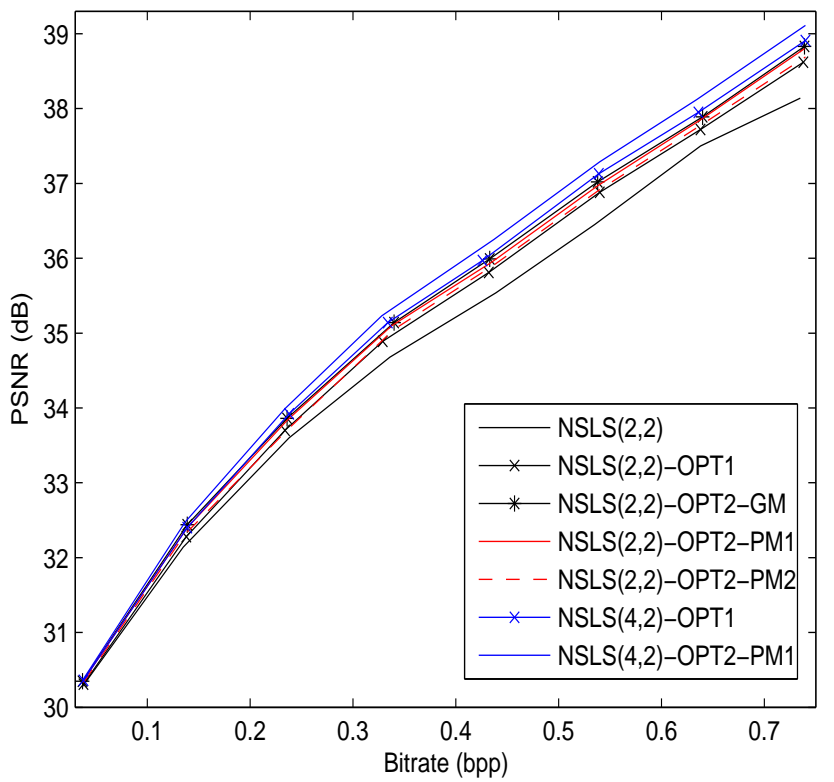

Figure 11: PSNR (in dB) versus the bitrate (bpp) after JPEG2000 progressive encoding for the "spot5-3" residual image. 


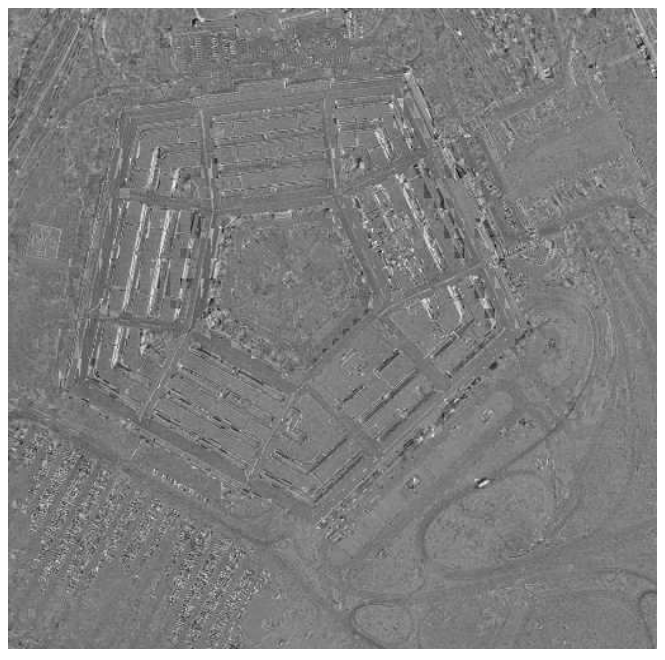

(a): Original residual image

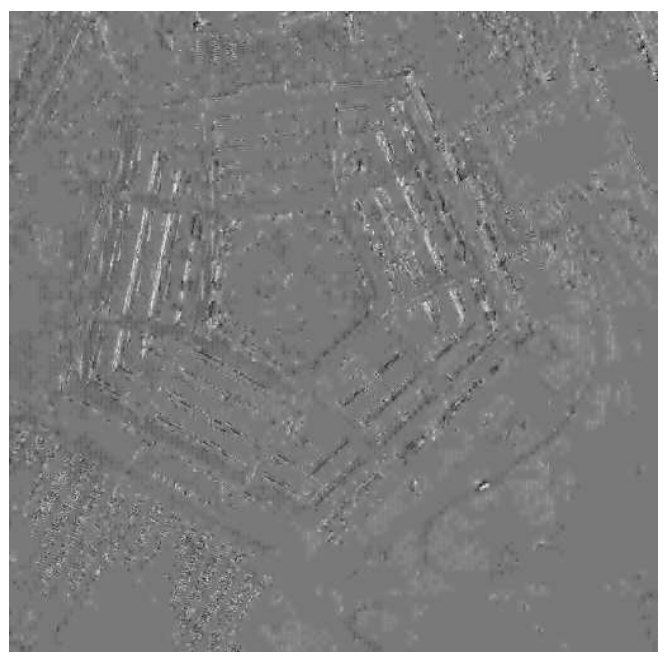

(c): PSNR $=29.65 \mathrm{~dB}, \mathrm{SSIM}=0.515$

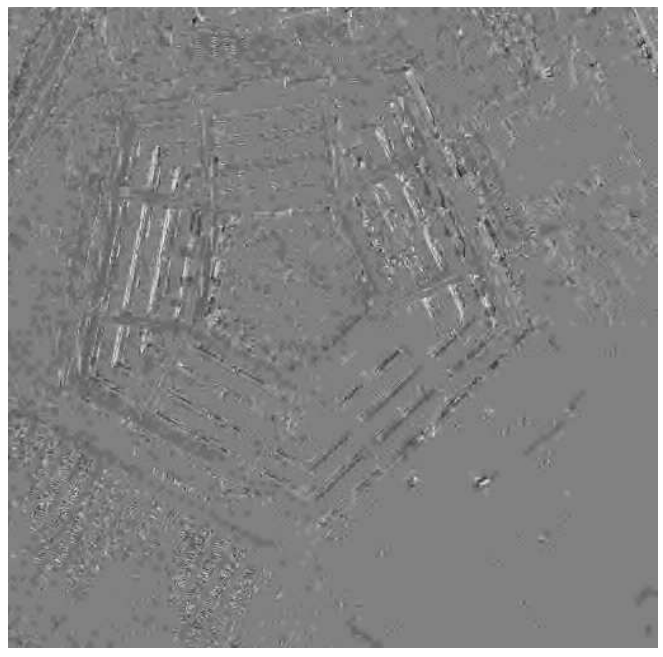

(b): $\mathrm{PSNR}=29.60 \mathrm{~dB}, \mathrm{SSIM}=0.484$

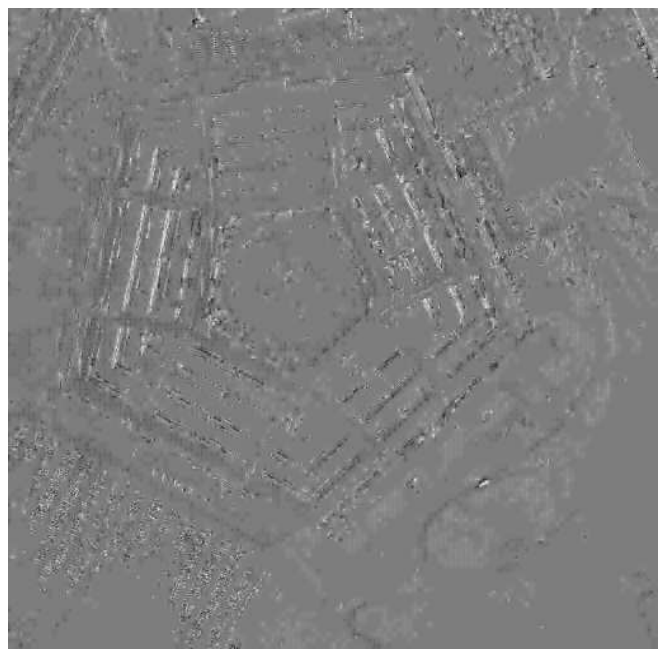

(d): PSNR $=29.87 \mathrm{~dB}, \mathrm{SSIM}=0.541$

Figure 12: The reconstructed "pentagone" residual image at 0.31 bpp using: (b) NSLS $(2,2)$ (c) NSLS(2,2)-OPT1 (d) NSLS(2,2)-OPT2-PM1.

A more exhaustive study was also conducted by applying the proposed optimization method to 10 still images and 10 stereo images downloaded from the databases mentioned at the beginning of this section. Figures 13 and 14 illustrate the average PSNR per-image 
in the case of still and stereo image coding.

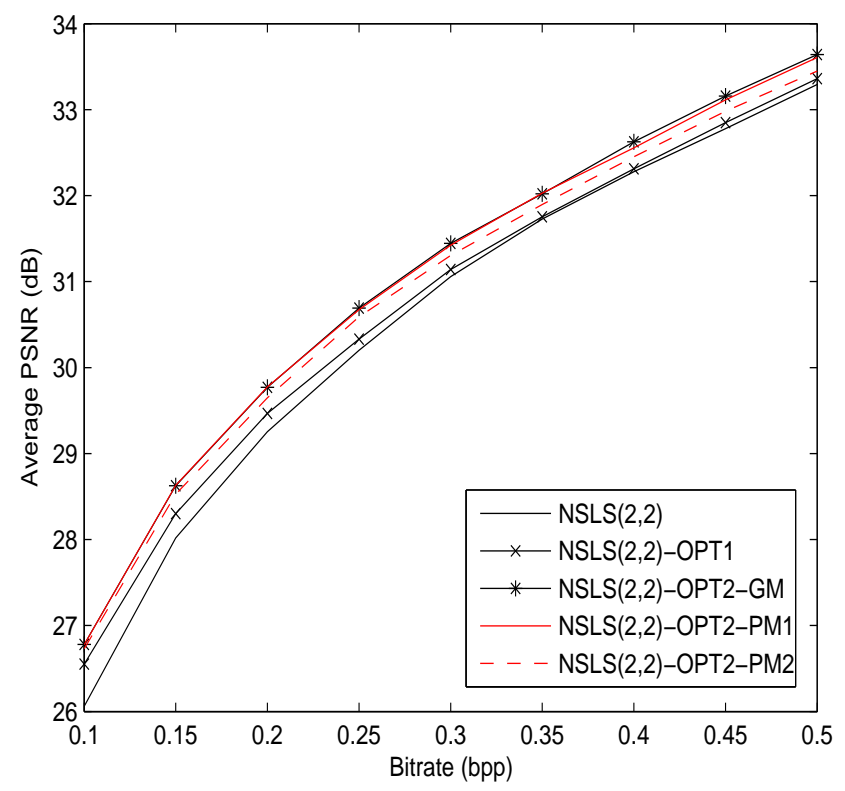

Figure 13: Average PSNR (in dB computed over 10 still images) versus the bitrate (bpp) after JPEG2000 progressive encoding.

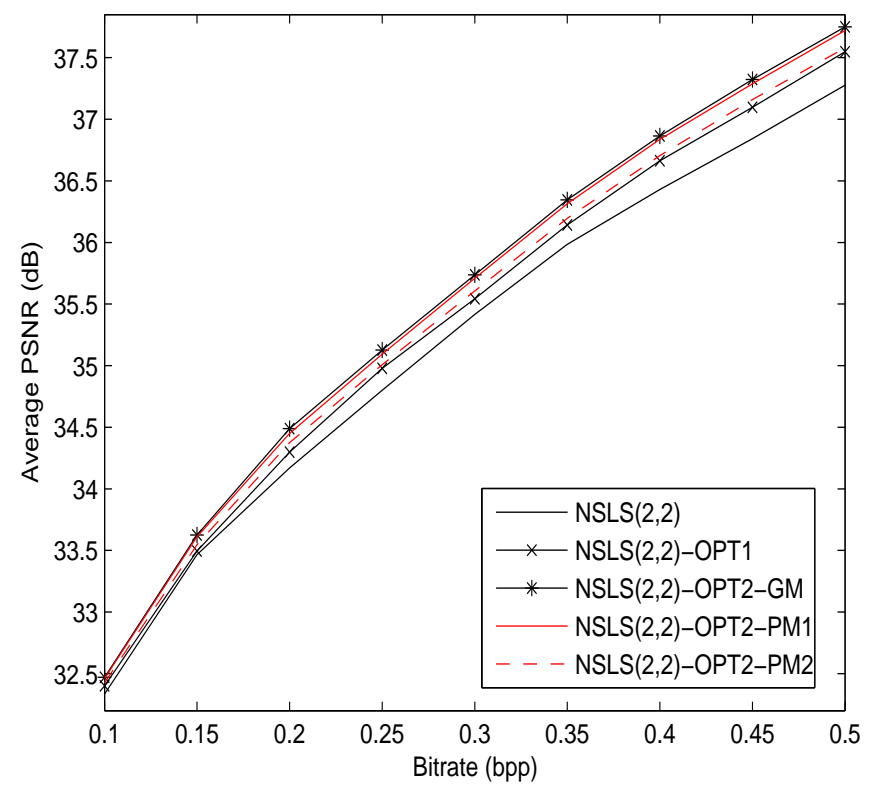

Figure 14: Average PSNR (in dB computed over 10 residual images) versus the bitrate (bpp) after JPEG2000 progressive encoding. 
All these results, obtained with monocular images and residual images of stereo pairs, confirm the effectiveness of our method in terms of reconstruction quality.

\section{Conclusions}

In this paper, we have exploited the flexibility offered by non separable lifting schemes to optimize their operators. A new criterion has been presented for the optimization of the update filter in this context. The proposed method adapts the filters to the contents of the input image while ensuring perfect reconstruction. A theoretical analysis in terms of the retained adaptation criterion was conducted in order to show the benefits that can be drawn from this optimization method. Furthermore, this study provides closed form expressions of the optimal filter coefficients which, due to their simplicity, can be exploited in the implementation process. Experimental results, carried out on still images and residual images of stereo pairs have illustrated the good performance in terms of bitrate and quality of reconstruction when optimizing both the prediction and the update filters. In future work, we plan to extend this optimization method to lifting schemes with more than two stages like the P-U-P structure and the P-U-P-U one.

\section{References}

[1] P. Vaidyanathan, Multirate systems and filter banks, Englewood Cliffs, NJ: Prentice Hall, 1992.

[2] J. Woods, Ed., Subband image coding, Nonuell, MA: Kluwer, 1991.

[3] K. Sayood, Introduction to Data Compression, Academic Press, San Diego, Californie, USA, 1996.

[4] D. Taubman and M. Marcellin, JPEG2000: Image compression fundamentals, standards and practice, Kluwer Academic Publisher, Boston, MA, USA, 2002.

[5] A. Benazza-Benyahia and J.-C. Pesquet, "A unifying framework for lossless and progressive image coding," Pattern Recognition, vol. 35, no. 3, pp. 627-638, November 2001.

[6] W. Sweldens, "The lifting scheme: A custom design of biorthogonal wavelets," J. Appl. Comput. Harmonic Anal., vol. 3, no. 2, pp. 186-200, April 1996.

[7] A. R. Calderbank, I. Daubechies, W. Sweldens and B.-L. Yeo, "Wavelet transforms that map integers to integers," J. Appl. Comput. Harmonic Anal., vol. 5, pp. 332-369, July 1998.

[8] O. N. Gerek and A. E. Cetin, "Adaptive polyphase subband decomposition structures for image compression," IEEE Transactions on Image Processing, vol. 9, no. 10, pp. 1649-1660, October 2000.

[9] M. Kaaniche, A. Benazza-Benyahia, B. Pesquet-Popescu and J.-C. Pesquet, "Vector lifting schemes for stereo image coding," IEEE Transactions on Image Processing, vol.18, no.11, pp. 2463-2475, November 2009. 
[10] N. V. Boulgouris and M. G. Strintzis, 'A family of wavelet-based stereo image coders," IEEE Trans. on Circuits and Systems for Video Technology, vol. 12, no. 10, pp. 898-903, October 2002.

[11] S. Wang and H. Chen, "An improved algorithm of motion compensation MPEG video compression," Int. Vehicle Electronics Conference, vol. 1, pp. 261-264, Changchun, China, 6-9 September 1999.

[12] T. Frajka and K. Zeger, "Residual image coding for stereo image compression," Optical Engineering, vol. 42, no. 1, pp. 182-189, January 2003.

[13] M. S. Moellenhoff and M. W. Maier, "Characteristics of disparity-compensated stereo image pair residuals," Signal Processing, Image Communications, vol. 14, no. 1-2, pp. 55-69, November 1998.

[14] A. Gouze, M. Antonini, M. Barlaud, and B. Macq, "Optimized lifting scheme for two-dimensional quincunx sampling images," IEEE International Conference on Image Processing, vol. 2, pp. 253258, Thessaloniki, Greece, 7-10 October 2001.

[15] A. Benazza-Benyahia, J.-C. Pesquet, J. Hattay, H. Masmoudi, "Block-based adaptive vector lifting schemes for multichannel image coding," Eurasip International Journal of Image and Video Processing (IJIVP), vol. 2007, no. 1, article ID 13421, 10 pages, January 2007.

[16] S. Sezen and A. Ertuzun, "2D four-channel perfect reconstruction filter bank realized with the 2D lattice filter structure," EURASIP Journal on Applied Signal Processing, vol. 2006, pp. 1-16, January 2006.

[17] S. Chokchaitam, "A non-separable two-dimensional LWT for an image compression and its theoretical analysis," Thammasat International Journal of Science and Technology, vol. 9, no. 1, pp. 35-43, January-March 2004.

[18] R. L. Claypoole, G. M. Davis, W. Sweldens and R. G. Baraniuk, "Nonlinear wavelet transforms for image coding via lifting," IEEE Transactions on Image Processing, vol. 12, no. 12, pp. 1449-1459, December 2003.

[19] O. N. Gerek and A. E. Cetin, "A 2D orientation-adaptive prediction filter in lifting structures for image coding," IEEE Transactions on Image Processing, vol. 15, no. 1, pp. 106-111, January 2006.

[20] N. V. Boulgouris and M. G. Strintzis, "Reversible multiresolution image coding based on adaptive lifting," IEEE International conference on Image Processing, no. 3, pp. 546-550, Kobe, Japan, 24-28 October 1999

[21] W. Ding, F. Wu, X. Wu, S. Li and H. Li, "Adaptive directional lifting-based wavelet transform for image coding," IEEE Trans. on Image Processing, vol. 10, no. 2, pp. 416-427, February 2007.

[22] C.-L. Chang and B. Girod, "Direction Adaptive discrete wavelet transform for image compression," IEEE Transactions on Image Processing, vol. 16, no. 5, pp. 1289-1302, May 2007.

[23] Y. Liu, K. N. Ngan, "Weighted adaptive lifting-based wavelet transform for image coding," IEEE Transactions on Image Processing, vol. 17, no. 4, pp. 500-511, April 2008.

[24] S. Mallat, "Geometrical grouplets," Applied and Computational Harmonic Analysis, vol. 26, no. 2, pp. 161-180, March 2009.

[25] J. Solé and P. Salembier, "Generalized lifting prediction optimization to lossless image compression," IEEE Signal Processing Letters, vol. 14, no. 10, pp. 695-698, October 2007.

[26] V. Chappelier and C. Guillemot, "Oriented wavelet transform for image compression and de- 
noising", IEEE Transactions on Image Processing, Vol. 15, No. 10, pp. 2892-2903, October 2006.

[27] G. Piella, B. Pesquet-Popescu and H. Heijmans, "Adaptive update lifting with a decision rule based on derivative filters," IEEE Signal Processing Letters, vol. 9, no. 10, pp. 329-332, October 2002.

[28] G. C. K. Abhayaratne, G. Piella, H. Heijmans and B. Pesquet-Popescu, "Adaptive integer to integer wavelet transforms using update lifting," In Wavelet Applications in Signal and Image Processing X, SPIE, vol. 5207, pp. 813-824, San Diego, CA, USA, 04 August 2003.

[29] G. C. K. Abhayaratne, "2D wavelet transforms with a spatially adaptive 2D low-pass filter," In Proc. of the 6th Nordic Signal Processing Symposium, Espoo, Finland, pp. 93-96, 9-11 June 2004.

[30] B. Pesquet-Popescu, "Two-stage adaptive filter bank", first filling date 1999/07/27, official filling number 99401919.8, European patent number EP1119911.

[31] A. Gouze, M. Antonini, M. Barlaud and B. Macq, "Design of signal-adapted multidimensional lifting schemes for lossy coding," IEEE Trans. on Image Processing, vol. 13, no. 12, pp. 1589-1603, December 2004.

[32] G. Quellec, M. Lamard, G. Gazuguel, B. Cochener and C. Roux, "Adaptive nonseparable wavelet transform via lifting and its application to content-based image retrieval," IEEE Trans. on Image Processing, vol. 19, no. 1, pp. 25-35, January 2010.

[33] D. Taubman, Adaptive, non-separable lifting transforms for image compression, in Proc. IEEE Int. Conf. Image Processing, Kobe, Japan, vol. 3, pp. 772-776, 24-28 October 1999.

[34] M. Kaaniche, J.-C. Pesquet, A. Benazza-Benyahia and B. Pesquet-Popescu, "Two-dimensional non separable adaptive lifting scheme for still and stereo image coding," IEEE International Conference on Acoustics, Speech and Signal Processing, 14-19 March 2010.

[35] S. Chabrier, B. Emile and C. Rosenberger, "Texture detection for image analysis", Springer-Verlag, vol. 3687/2005, pp. 455-463, Berlin, October 2005.

[36] J. M. Shapiro, "Embedded image coding using zerotrees of wavelet coefficients," IEEE Transactions on Signal Processing, vol. 41, no. 12, pp. 3445-3462, December 1993.

[37] A. Said and W.A. Pearlman, "A new fast and efficient image codec based on set partitionning in hierarchical trees," IEEE Transactions on Circuits and Systems for Video Technology, vol. 6, pp. 243-250, June 1996.

[38] D. Taubman, "High performance scalable image compression with EBCOT," IEEE Transactions on Image Processing, vol. 9, no. 7, pp. 1158-1170, July 2000.

[39] S.-T. Hsiang and J. W. Woods, "Embedded image coding using zeroblocks of subband/wavelet coefficients and context modeling," IEEE International Symposium on Circuits and Systems, vol. 3, pp. 662-665, Geneva, Switzerland, May 2000.

[40] Z. Wang, A. C. Bovik, H. R. Sheikh and E. P. Simoncelli, "Image quality assessment: From error visibility to structural similarity," IEEE Transactions on Image Processing, vol. 13, no. 4, pp. 600612, April 2004. 